MITP/15-019

\title{
Charge transport and vector meson dissociation across the thermal phase transition in lattice QCD with two light quark flavors
}

\author{
Bastian B. Brandt ${ }^{1}$, Anthony Francis ${ }^{2}$, Benjamin Jäger ${ }^{3}$ and Harvey B. Meyer ${ }^{4}$ \\ ${ }^{1}$ Institut für theoretische Physik, Universität Regensburg, D-93040 Regensburg, Germany \\ ${ }^{2}$ Department of Physics $\&$ Astronomy, York University, \\ 4700 Keele St, Toronto, ON M3J 1P3, Canada \\ ${ }^{3}$ Department of Physics, College of Science, \\ Swansea University, SA2 8PP Swansea, United Kingdom \\ ${ }^{4}$ PRISMA Cluster of Excellence, Institut für Kernphysik and Helmholtz Institut Mainz, \\ Johannes Gutenberg-Universität Mainz, D-55099 Mainz, Germany
}

(Dated: December 23, 2015)

\begin{abstract}
We compute and analyze correlation functions in the isovector vector channel at vanishing spatial momentum across the deconfinement phase transition in lattice QCD. The simulations are carried out at temperatures $T / T_{c}=0.156,0.8,1.0,1.25$ and 1.67 with $T_{c} \simeq 203 \mathrm{MeV}$ for two flavors of Wilson-Clover fermions with a zero-temperature pion mass of $\simeq 270 \mathrm{MeV}$. Exploiting exact sum rules and applying a phenomenologically motivated ansatz allows us to determine the spectral function $\rho(\omega, T)$ via a fit to the lattice correlation function data. From these results we estimate the electrical conductivity across the deconfinement phase transition via a Kubo formula and find evidence for the dissociation of the $\rho$ meson by resolving its spectral weight at the available temperatures. We also apply the Backus-Gilbert method as a model-independent approach to this problem. At any given frequency, it yields a local weighted average of the true spectral function. We use this method to compare kinetic theory predictions and previously published phenomenological spectral functions to our lattice study.
\end{abstract}

\section{INTRODUCTION}

Strongly interacting matter at temperatures below $100 \mathrm{MeV}$ is thought to consist of hadronic quasiparticles to a good approximation. At sufficiently high temperatures, perhaps of several $\mathrm{GeV}$, one expects quarks and gluons to be the relevant degrees of freedom instead. The stages between the two regimes are the subject of vast experimental programs based on colliding heavy ions and of many theoretical studies (see for instance [1], chapter D). The broad interest partly stems from the fact that matter in the early universe underwent a thermal transition between the two phases. This transition is known from lattice simulations to be a continuous crossover rather than a sharp phase transition [2].

Hard probes, observables which, although produced in the thermal medium, immediately decouple from it, are especially interesting in this context. With no subsequent interaction after their production, they are among the best observables to study the thermal medium both in experiment [3] and theory. For a quark gluon plasma a good probe is the production rate of lepton pairs, since they experience only electroweak interactions after they are created and act as essentially freely propagating particles. At the same time the underlying production mechanism is highly complicated due to the strong interactions of the constituents of the thermal system. The dilepton production rates for a system in thermal equilibrium are proportional to the thermal vector spectral function. The latter encodes the information on the excitation spectrum and transport processes in the medium (see for instance [4]).

The hadron resonance gas (HRG) model, which is based on the idea that the static properties of strongly interacting matter in the low-temperature phase are given by the sum of the contributions of non-interacting hadron species, gives an economic description of particle yields in heavy-ion col- 
lisions (see the recent [5], 6] and references therein) and provides a good estimate of the pressure and charge fluctuations determined in lattice calculations [7 9]. However, the thermal quasiparticles could have different properties, for instance different dispersion relations [10, 11, from the hadrons at $T=0$. One of our goals here is to probe to what extent the transport of charge can be described by the HRG model.

In a strongly coupled regime, no general computational method to address thermal real-time phenomena is known. Interesting insights have been gained via the gauge/gravity correspondence (see for instance [12]). Lattice QCD provides a way to compute correlation functions in the Matsubara (or Euclidean) formalism. These correlation functions are related to spectral functions $\rho(\omega, T)$ via dispersion relations, as a consequence they provide information on the latter. However, obtaining information that is more local in the frequency $\omega$ than $\Delta \omega \sim T$ requires highly accurate calculations of the Euclidean correlation functions. In recent years, the quality of this type of lattice calculations has steadily improved [13, 20].

In the following we present a study of isovector vector correlation functions across the deconfinement phase transition on large isotropic lattice ensembles using Wilson-Clover fermions. After presenting the theoretical background to our calculations (section II) and our numerical framework (section III), we apply an ansatz for the spectral functions and fit its parameters to the lattice data (section IV A). Thereby we estimate the electrical conductivity and the spectral weight of the $\rho$ meson for a range of temperatures. In section IVB, we apply the model-independent BackusGilbert method [21] to obtain a 'filtered' spectral function, which is a local average of the genuine spectral function around a given frequency. In this way we obtain a means to directly compare HRG predictions and previously published phenomenological spectral functions [22] with lattice data (section V). The phenomenological implications of our calculation are discussed in section VI. The article ends with a summary of the lessons we learnt from the lattice calculations and an outlook on how further insight could be gained on the thermal spectral functions.

\section{DEFINITIONS AND THEORY PREDICTIONS}

In this section we collect the necessary definitions and review (mostly known) facts about correlation functions and the associated spectral functions of the vector current.

\section{A. Definitions}

Our primary observables are the Euclidean-time correlators of the conserved vector current,

$$
G_{\mu \nu}(\tau, T)=\int d^{3} x\left\langle J_{\mu}(\tau, \vec{x}) J_{\nu}(0)^{\dagger}\right\rangle .
$$

The expectation values are taken with respect to the equilibrium density matrix $e^{-\beta H} / Z(\beta)$, where $\beta \equiv 1 / T$ is the inverse temperature, and the Euclidean-time evolution of operators is given by $\mathcal{O}(\tau)=e^{H \tau} \mathcal{O} e^{-H \tau}$. For now we leave the flavor structure of the current unspecified and return to this aspect in the next subsection. We define the quark number susceptibility as

$$
\chi_{s}=\beta \int d^{3} x\left\langle J_{0}(\tau, \vec{x}) J_{0}(0)\right\rangle, \quad \tau \neq 0 .
$$

The Euclidean correlators have the spectral representation

$$
G_{\mu \nu}(\tau, T) \stackrel{\mu=\nu}{=} \int_{0}^{\infty} \frac{d \omega}{2 \pi} \rho_{\mu \nu}(\omega, T) \frac{\cosh [\omega(\beta / 2-\tau)]}{\sinh (\omega \beta / 2)},
$$


where the spectral function is defined (for a general spatial momentum $\vec{p}$ ) as

$$
\rho_{\mu \nu}(\omega, \vec{p}, T)=\int d t d^{3} x e^{i \omega \cdot t-i \vec{p} \cdot \vec{x}}\left\langle\left[J_{\mu}(t, \vec{x}), J_{\nu}(0)^{\dagger}\right]\right\rangle, \quad \mathcal{O}(t) \equiv e^{i H t} \mathcal{O} e^{-i H t} .
$$

For a given function $\rho\left(\omega, T^{\prime}\right)$, the reconstructed correlator is defined as

$$
G_{\text {rec }}\left(\tau, T ; T^{\prime}\right) \equiv \int_{0}^{\infty} \frac{d \omega}{2 \pi} \rho\left(\omega, T^{\prime}\right) \frac{\cosh [\omega(\beta / 2-\tau)]}{\sinh (\omega \beta / 2)} .
$$

It can be interpreted as the Euclidean correlator that would be realized at temperature $T$, if the spectral function did not change between temperature $T$ and $T^{\prime}$. For $T^{\prime}=0$ it can be directly obtained from the zero-temperature Euclidean correlator via 23.

$$
G_{\mathrm{rec}}(\tau, T) \equiv G_{\mathrm{rec}}(\tau, T ; 0)=\sum_{m \in \mathbb{Z}} G(|\tau+m \beta|, T=0) .
$$

\section{B. On the flavor structure of the current}

Unless otherwise stated, we consider two-point functions of the isospin current ${ }^{1}$

$$
J_{\mu}(x) \equiv \frac{1}{\sqrt{2}}\left(\bar{u}(x) \gamma_{\mu} u(x)-\bar{d}(x) \gamma_{\mu} d(x)\right)
$$

in QCD with two light degenerate flavors of quarks, and the associated spectral function $\rho_{\mu \nu}(\omega, T)$.

Many phenomenological observables are related to the electromagnetic current

$$
J_{\mu}^{\mathrm{em}}=\sum_{f} Q_{f} \bar{q}_{f} \gamma_{\mu} q_{f}
$$

with $Q_{u}=2 / 3, Q_{d}=-1 / 3$ etc. and the associated spectral function $\rho_{\mu \nu}^{\mathrm{em}}(\omega, \vec{p}, T)$. In two-flavor QCD, we can decompose the electromagnetic current into the following sum of isovector and isoscalar components,

$$
J_{\mu}^{\mathrm{em}}=\frac{1}{\sqrt{2}} J_{\mu}+\frac{1}{2} J_{\mu}^{\mathrm{B}}
$$

where

$$
J_{\mu}^{\mathrm{B}}=\frac{1}{3}\left(\bar{u}(x) \gamma_{\mu} u(x)+\bar{d}(x) \gamma_{\mu} d(x)\right)
$$

is the baryon current. Since in this work we compute only the two-point function of $J_{\mu}$, the question poses itself as to whether the two-point function of $J_{\mu}^{\mathrm{em}}$ can be approximated with the available information. In the vacuum, this question has been addressed recently [24, 25]. It turns out that, as far as the spectral function is concerned, the appropriate approximation depends on the frequency. At sufficiently high frequencies, a decomposition of the two-point function of $J_{\mu}^{\mathrm{em}}$ in terms of a quark-line connected contribution and a disconnected contribution is most useful, since the latter is of order $\alpha_{s}^{3}$ in perturbative QCD. When the quark-line disconnected contribution to the two-point function of $J_{\mu}^{\mathrm{em}}$ can be neglected, we have

$$
\left.\rho_{\mu \nu}^{\mathrm{em}}(\omega, \overrightarrow{0}, T)\right|_{n_{f}=2} \simeq C_{\mathrm{em}} \rho_{\mu \nu}(\omega, T), \quad C_{\mathrm{em}}=\sum_{f=u, d} Q_{f}^{2}=5 / 9 .
$$

\footnotetext{
${ }^{1}$ Note that in this normalization, all two-point functions of the current are a factor 2 larger than the isovector contributions to the two-point functions of the electromagnetic current.
} 
On the other hand, isospin symmetry implies that the two-point function of $J_{\mu}^{\mathrm{em}}$ is given by the sum of the two-point function of $\frac{1}{\sqrt{2}} J_{\mu}$ and of the two-point function of $\frac{1}{2} J_{\mu}^{\mathrm{B}}$. For $\omega \lesssim m_{\omega}$, where $m_{\omega}$ is the mass of the (isoscalar vector) $\omega$ meson, the isoscalar two-point function is negligible compared to the isovector contribution; in fact, the corresponding contribution to the spectral function is exactly zero for $0 \leq \omega \leq 3 m_{\pi}$. Therefore, in this low-frequency regime the appropriate approximation is

$$
\rho_{\mu \nu}^{\mathrm{em}}(\omega, \overrightarrow{0}, T) \simeq \frac{1}{2} \rho_{\mu \nu}(\omega, T), \quad 0 \leq \omega \lesssim m_{\omega}
$$

One may ask whether any of the two presented approximations still holds at non-zero temperature. At sufficiently high temperatures, when the relevant degrees of freedom are quarks and gluons, we expect that the approximation given in Eq. (11) is applicable at all frequencies. In the low-temperature phase, we expect the same approximation to hold as for zero temperature, namely Eq. (12) for $\omega \lesssim m_{\omega}$ and Eq. (11) at higher frequencies. This expectation is based in particular on the observation that only baryons contribute to the transport peak in the spectral function associated with the current $J_{\mu}^{\mathrm{B}}$ (see section II E below); this transport peak therefore carries far less spectral weight than the transport peak in the isovector channel, since the latter receives contributions from pions, $\rho$ mesons etc.

\section{General properties of spectral functions and their relation to observables}

In the thermodynamic limit, the subtracted vector spectral function obeys the exact sum rule (see [26] sec. 3.2),

$$
\int_{-\infty}^{\infty} \frac{d \omega}{\omega} \Delta \rho(\omega, T)=0, \quad \Delta \rho(\omega, T) \equiv \rho_{i i}(\omega, T)-\rho_{i i}(\omega, 0)
$$

The diffusion constant $D$ is given by a Kubo formula in terms of the low-frequency behavior of the spectral function,

$$
D=\frac{1}{6 \chi_{s}} \lim _{\omega \rightarrow 0} \frac{\rho_{i i}(\omega, T)}{\omega} .
$$

The electrical conductivity in two flavor QCD is given by

$$
\sigma_{\mathrm{el}}=\frac{1}{6} \lim _{\omega \rightarrow 0} \frac{\rho_{i i}^{\mathrm{em}}(\omega, \overrightarrow{0}, T)}{\omega} \simeq C_{e m} D \chi_{s}
$$

In the above equation, disconnected diagrams have been neglected. More generally, the electromagnetic spectral function determines the differential production rate of lepton-antilepton pairs per unit volume of the thermal system,

$$
\frac{d N_{l^{+} l^{-}}}{d \omega d^{3} p}=C_{\mathrm{em}} \frac{\alpha_{\mathrm{em}}^{2}}{6 \pi^{3}} \frac{\rho_{\mu \mu}^{\mathrm{em}}(\omega, \vec{p}, T)}{\left(\omega^{2}-\vec{p}^{2}\right)\left(e^{\omega / T}-1\right)} .
$$

\section{Non-interacting limits of the spectral functions}

There are two simple limits, in which the spectral function can be determined analytically. For non-interacting massive quarks in the fundamental representation of the $\mathrm{SU}\left(N_{c}\right)$ color group, the 
vector spectral function is given by $\left.\right|^{2}$

$$
\rho_{i i}(\omega, T)=2 \pi \chi_{s}\left\langle v^{2}\right\rangle \omega \delta(\omega)+\frac{N_{c}}{2 \pi} \theta(\omega-2 m)\left[1-\frac{4 m^{2}}{\omega^{2}}\right]^{\frac{1}{2}}\left[1+\frac{2 m^{2}}{\omega^{2}}\right] \omega^{2} \tanh (\omega \beta / 4) .
$$

The sum rule Eq. (13) is verified by this spectral function. On the other hand, at low temperatures and at low frequency, we expect pions to be the relevant degrees of freedom. In the theory of non-interacting pions, the third isospin component of the vector current is represented by $J_{\mu}=$ $\sqrt{2}\left(\pi^{1} \partial_{\mu} \pi^{2}-\pi^{2} \partial_{\mu} \pi^{1}\right)=\sqrt{2} i\left(\pi^{+} \partial_{\mu} \pi^{-}-\pi^{-} \partial_{\mu} \pi^{+}\right)$. The spectral function reads

$$
\rho_{i i}(\omega, T)=2 \pi \chi_{s}\left\langle v^{2}\right\rangle \omega \delta(\omega)+\frac{1}{4 \pi} \theta\left(\omega-2 m_{\pi}\right)\left[1-\frac{4 m_{\pi}^{2}}{\omega^{2}}\right]^{\frac{3}{2}} \frac{\omega^{2}}{\tanh (\omega \beta / 4)} .
$$

For free quarks, the susceptibility $\chi_{s}$ and the mean squared transport velocity $\left\langle v^{2}\right\rangle$ are given by,

$$
\begin{aligned}
\chi_{s} & =4 N_{c} \beta \int \frac{d^{3} \vec{p}}{(2 \pi)^{3}} f_{\vec{p}}^{F}\left(1-f_{\vec{p}}^{F}\right), \\
\chi_{s}\left\langle v^{2}\right\rangle & =4 N_{c} \beta \int \frac{d^{3} \vec{p}}{(2 \pi)^{3}} f_{\vec{p}}^{F}\left(1-f_{\vec{p}}^{F}\right) \frac{\vec{p}^{2}}{E_{\vec{p}}^{2}},
\end{aligned}
$$

while for free pions the expressions are ${ }^{3}$

$$
\begin{gathered}
\chi_{s}=4 \beta \int \frac{d^{3} \vec{p}}{(2 \pi)^{3}} f_{\vec{p}}^{B}\left(1+f_{\vec{p}}^{B}\right), \\
\chi_{s}\left\langle v^{2}\right\rangle=4 \beta \int \frac{d^{3} \vec{p}}{(2 \pi)^{3}} f_{\vec{p}}^{B}\left(1+f_{\vec{p}}^{B}\right) \frac{\vec{p}^{2}}{E_{\vec{p}}^{2}} .
\end{gathered}
$$

The energies in Eqs. $19-122$ are respectively the free energies of quarks $E_{\vec{p}}=\sqrt{\vec{p}^{2}+m^{2}}$ and pions $E_{\vec{p}}=\sqrt{\vec{p}^{2}+m_{\pi}^{2}}$, while $f_{\vec{p}}^{B / F}=1 /\left[e^{\beta E_{\vec{p}}} \mp 1\right]$ are the Bose-Einstein and Fermi-Dirac distributions. The mean square velocity is unity for massless particles and $3 T / m$ for non-relativistic particles. The susceptibility of free particles has simple expressions in the high-temperature limit

$$
\chi_{s} \stackrel{T \rightarrow \infty}{=}\left\{\begin{array}{c}
\left(N_{c} / 3\right) T^{2} \\
(2 / 3) T^{2}
\end{array}\right.
$$

and in the low-temperature limit

$$
\chi_{s} \stackrel{T \rightarrow 0}{=}\left\{\begin{array}{c}
\frac{\sqrt{2}}{\pi^{3 / 2}} T^{1 / 2} m^{3 / 2} e^{-\beta m} \\
N_{c} \frac{\sqrt{2}}{\pi^{3 / 2}} T^{1 / 2} m^{3 / 2} e^{-\beta m}
\end{array},\right.
$$

where the upper cases correspond to quarks and the lower ones to pions.

Beyond the strict non-interacting theory, weak coupling kinetic theory predicts the presence of a narrow transport peak in the spectral function at $\omega=0$, whose width and height are related to the properties of the quasi-particles. Introducing a separation scale $\Lambda$ between the transport time scale and the thermal time-scale, the area under the transport peak is, to leading order, preserved by the interactions [29],

$$
\mathcal{A}(\Lambda)=\int_{-\Lambda}^{\Lambda} \frac{d \omega}{2 \pi} \frac{\rho_{i i}(\omega, T)}{\omega}=\chi_{s}\left\langle v^{2}\right\rangle
$$

and the width of the transport peak becomes finite.

\footnotetext{
${ }^{2}$ At large frequencies the radiative corrections $\left(1+\alpha_{s} / \pi+\ldots\right)$ to the coefficient of the $\omega^{2}$ term are temperature independent and known to order $\alpha_{s}^{4}$ [27] (for quark mass effects in the vacuum, see [28]).

3 Note, the pion contribution to the susceptibility of the electric charge $Q$ is $1 / 2$ times the right-hand side of Eq. 21.
} 


\section{E. Kinetic theory and the hadron resonance gas}

We now explore the assumption that the thermal system in the low-temperature phase is well approximated by a gas of weakly interacting hadrons. In [20], we estimated that the area under the $\rho$ resonance peak in the vacuum spectral function $\rho_{i i}(\omega, 0)$ was

$$
\int_{0}^{1 \mathrm{GeV}} \frac{d \omega}{\pi} \frac{\rho_{i i}(\omega, 0)}{\omega}=0.114 \mathrm{GeV}^{2} .
$$

As we shall see shortly, the area under the transport peak represents no more than a $\sim 5 \%$ effect in comparison to the spectral weight of the $\rho$ meson 4 .

The hadron resonance gas describes the thermodynamic properties and the quark number susceptibilities of the low-temperature phase rather well. Assuming a transport peak exists in $\rho_{i i}(\omega, T)$, it is interesting to ask whether the area under the peak is consistent with this picture. It is straightforward to compute the sum of the mesonic and baryonic contributions, e.g. $\chi_{s}\left\langle v^{2}\right\rangle=\left(\chi_{s}\left\langle v^{2}\right\rangle\right)_{\text {mesons }}+\left(\chi_{s}\left\langle v^{2}\right\rangle\right)_{\text {baryons }}$,

$$
\begin{gathered}
\left(\chi_{s}\left\langle v^{2}\right\rangle\right)_{\text {mesons }}=\frac{2 \beta}{3} \sum_{\text {multiplets }}(2 J+1) I(I+1)(2 I+1) \int \frac{d^{3} \vec{p}}{(2 \pi)^{3}} f_{\vec{p}}^{B}\left(1+f_{\vec{p}}^{B}\right) \frac{\vec{p}^{2}}{E_{\vec{p}}^{2}}, \\
\left(\chi_{s}\left\langle v^{2}\right\rangle\right)_{\text {baryons }}=\frac{4 \beta}{3} \sum_{\text {multiplets }}(2 J+1) I(I+1)(2 I+1) \int \frac{d^{3} \vec{p}}{(2 \pi)^{3}} f_{\vec{p}}^{F}\left(1-f_{\vec{p}}^{F}\right) \frac{\vec{p}^{2}}{E_{\vec{p}}^{2}} .
\end{gathered}
$$

The sums are carried out over the multiplet 5 of spin $J$ and isospin $I$. The corresponding expressions for $\left(\chi_{s}\right)_{\text {mesons }}$ and $\left(\chi_{s}\right)_{\text {baryons }}$ are identical respectively to Eqs. (27) and (28), except for the absence of the factor $\frac{\vec{p}^{2}}{E_{\vec{p}}^{2}}$ in the integrand. Expanding the factor $f(1 \pm f)$ in a Taylor series in $e^{-\beta E_{\vec{p}}}$, one can express it in terms of a sum of Bessel functions (see for instance the expressions in [7]). At physical quark masses in two-flavor QCD, we thus obtain the HRG estimates (more details on our implementation of the HRG model are given in section $\mathrm{VA}$

$$
\chi_{s}\left\langle v^{2}\right\rangle / \mathrm{GeV}^{2}=\left\{\begin{array}{cc}
0.00236 & T=100 \mathrm{MeV} \\
0.0042 & T=120 \mathrm{MeV} \\
0.0069 & T=140 \mathrm{MeV} \\
0.0111 & T=160 \mathrm{MeV}
\end{array}\right.
$$

The pions dominate this quantity up to quite high temperatures: for instance, at $T=140 \mathrm{MeV}$, they still contribute $90 \%$ of the total value. We remark that combining the exact sum rule Eq. (13) with the kinetic sum rule Eq. 25, we have the property

$$
\int_{\Lambda}^{\infty} \frac{d \omega}{\pi} \frac{\Delta \rho(\omega, T)}{\omega}=-\chi_{s}\left\langle v^{2}\right\rangle
$$

Since the values in Eq. (29) are much smaller than the area under the zero-temperature spectral function up to $\omega=1 \mathrm{GeV}$ given in Eq. (26), the spectral weight above the threshold $\omega=2 m_{\pi}$ is not substantially modified by thermal effects as long as the HRG remains a good approximation for evaluating $\chi_{s}\left\langle v^{2}\right\rangle$. The HRG model is in that sense self-consistent.

\footnotetext{
${ }^{4}$ From the point of view of large- $N_{c}$ counting rules, this is not surprising, since the spectral weight of the $\rho$ meson is of order $N_{c}$, while the area under the transport peak is of order $N_{c}^{0}$.

${ }^{5}$ The additional factor of 2 present in the baryon case accounts for antiparticles; in the meson case, the antiparticles are already included by summing $\sum_{I_{3}=-I}^{I} I_{3}^{2}=\frac{1}{3} I(I+1)(2 I+1)$.
} 


\section{NUMERICAL SETUP}

We present a study of thermal isovector vector two-point functions for temperatures $T / T_{c}=$ $0.16,0.8,1.0,1.25$ and 1.67 in two-flavor QCD. We vary the temperature by increasing the number of lattice sites in the time directions at fixed bare parameters, a method known as the 'fixed-scale' approach. The mass of the degenerate quark doublet corresponds to a zero-temperature pion mass of $\simeq 270 \mathrm{MeV}$ [30]. The data presented here is thus a natural extension of the calculation at $T / T_{c}=1.25$ presented in [20]. The gauge action is the standard Wilson plaquette action [31, while the fermions are implemented via the $\mathrm{O}(a)$ improved Wilson discretization with the non-perturbatively determined clover coefficient $c_{\mathrm{Sw}}$ [32]. All configurations are generated using the MP-HMC algorithm [33, 34] in the implementation of Marinkovic and Schaefer [35] based on Lüscher's DD-HMC package [36].

\begin{tabular}{|c|c|c|c|}
\hline $6 / g_{0}^{2}$ & 5.50 & $m_{\pi}[\mathrm{MeV}]$ & $\simeq 270$ \\
\hline$\kappa$ & 0.13671 & & $0.768(5)$ \\
\hline$c_{S W}$ & 1.751496 & $a[\mathrm{fm}]$ & $0.0486(4)(5)$ \\
\hline$N_{s}^{3} \times N_{\tau}$ & $64^{3} \times 128$ & $N_{\mathrm{conf}}^{\mathrm{vac}}$ & 137 \\
\hline$T^{\mathrm{vac}}[\mathrm{MeV}]$ & $32(6)$ & $N_{\mathrm{src}}^{\mathrm{vac}}$ & 16 \\
\hline$N_{s}^{3} \times N_{\tau}$ & $64^{3} \times 24$ & $N_{s}^{3} \times N_{\tau}$ & $64^{3} \times 20$ \\
\hline$T[\mathrm{MeV}]$ & $169(3)$ & $T[\mathrm{MeV}]$ & $203(4)$ \\
\hline$N_{\text {conf }}$ & 360 & $N_{\text {conf }}$ & 311 \\
\hline$N_{\mathrm{src}}$ & 64 & $N_{\mathrm{src}}$ & 64 \\
\hline$N_{s}^{3} \times N_{\tau}$ & $64^{3} \times 16$ & $N_{s}^{3} \times N_{\tau}$ & $64^{3} \times 12$ \\
\hline$T[\mathrm{MeV}]$ & $254(4)$ & $T[\mathrm{MeV}]$ & $338(5)$ \\
\hline$N_{\text {conf }}$ & 313 & $N_{\text {conf }}$ & 262 \\
\hline$N_{\text {src }}$ & 65 & $N_{\mathrm{src}}$ & 65 \\
\hline
\end{tabular}

TABLE I. The top left block shows the bare lattice parameters, see also [20, 37, 38. The top right block summarizes the pion mass, the vector renormalization constant 39] and the lattice spacing [37. The middle and bottom blocks contain more specific information on the $N_{\tau}=128,24,20,16$ and 12 lattice calculations, such as their corresponding temperatures, their number of configurations and the number of source positions used to calculate the correlation functions.

The spatial size of the ensembles is $N_{s}=64$ with periodic boundary conditions and the temporal extents are $N_{\tau}=128,24,20,16$ and 12. The $N_{\tau}=16$ ensemble was first presented in [20] and has subsequently been analyzed in 40 42. All ensembles are calculated at fixed bare parameters, whereby the lattice spacing is $a=0.0486(4)(5) \mathrm{fm}$ [37] and $m_{\pi} L=4.2$. With $T=1 /\left(N_{\tau} a\right)$ the ensembles correspond to the temperatures $T=169(3) \mathrm{MeV}\left(N_{\tau}=24\right), T=203(4) \mathrm{MeV}\left(N_{\tau}=20\right)$, $T=254(4) \mathrm{MeV}\left(N_{\tau}=16\right)$ and $T=338(5) \mathrm{MeV}\left(N_{\tau}=12\right)$. Based on preliminary results on the pseudo-critical temperature $T_{c}$ of the crossover from the hadronic to the high-temperature phase [38, 43], the temperatures can also be expressed in terms of $T_{c}$ by $T / T_{c} \approx 0.8,1.0,1.25$ and 1.67. In addition, we have updated our vacuum correlation function at $N_{\tau}=128$, i.e. $T=$ $32(6) \mathrm{MeV}$, by significantly increasing the statistics. We refer to this as the 'vacuum' ensemble.

In contrast to [20], we implement the vector correlation function on the lattice as a mixed 
correlator between the local and the conserved current,

$$
\begin{aligned}
& G_{i i}^{\mathrm{bare}}\left(\tau, g_{0}, T\right)=-a^{3} \sum_{i=1}^{3} \sum_{\vec{x}}\left\langle J_{i}^{c}(\tau, \vec{x}) J_{i}^{\ell}(0)\right\rangle, \\
& G_{00}^{\mathrm{bare}}\left(\tau, g_{0}, T\right)=-a^{3} \sum_{\vec{x}}\left\langle J_{0}^{c}(\tau, \vec{x}) J_{0}^{\ell}(0)\right\rangle,
\end{aligned}
$$

where

$$
\begin{aligned}
& J_{\mu}^{\ell}(x)=\frac{1}{\sqrt{2}} \bar{q}(x) \gamma_{\mu} \tau^{3} q(x), \\
& J_{\mu}^{c}(x)=\frac{1}{2 \sqrt{2}}\left(\bar{q}(x+a \hat{\mu})\left(1+\gamma_{\mu}\right) U_{\mu}^{\dagger}(x) \tau^{3} q(x)-\bar{q}(x)\left(1-\gamma_{\mu}\right) U_{\mu}(x) \tau^{3} q(x+a \hat{\mu})\right) .
\end{aligned}
$$

Here $U_{\mu}(x)$ are the gauge links, $q$ represents a doublet of mass-degenerate quark fields and $\tau^{3}$ the diagonal Pauli matrix acting on the flavor indices. The doublet can be understood as the $(\mathrm{u}, \mathrm{d})$ quarks and are treated fully dynamically in this calculation. To achieve a precision at the $\lesssim 1 \%$ level we supplement the source at position $x_{\mathrm{src}}=(0,0,0,0)$ with additional $N_{\mathrm{src}}=64$ randomly chosen source positions in the lattice four-volume in order to increase statistics by exploiting the translational invariance of the system. The correlators are renormalized via

$$
G_{\mu \nu}(\tau, T)=Z_{V}\left(g_{0}\right) G_{\mu \nu}^{\text {bare }}\left(\tau, g_{0}, T\right)
$$

with the non-perturbative value of $Z_{V}=0.768(5)$ [39]. Here, our primary goal is to carry out the analysis on a single lattice spacing. We therefore have not included $\mathrm{O}(a)$ contributions from the improvement term proportional to the derivative of the antisymmetric tensor operator [44, 45. Also a quark-mass dependent improvement term $\left(1+b_{V}\left(g_{0}\right) a m_{q}\right)$ [45] was neglected. These contributions should eventually be included to ensure a smooth scaling behavior as the continuum limit is taken. The parameters of the lattice ensembles are collected in Tab. I. In addition all results for the correlators are given in Tab. VII and Tab. VIII in Appendix A. The covariance matrices of these data sets are provided online on the arXiv [46].

The reconstructed correlator $G_{\mathrm{rec}}(\tau, T)$ can be straightforwardly computed when the ratio of vacuum and target temperature $N_{\tau}^{\mathrm{vac}} / N_{\tau}^{\text {target }}$ is an integer, which is the case for the $N_{\tau}^{\text {target }}=16$ ensemble, as the ratio is $N_{\tau}^{\mathrm{vac}} / N_{\tau}^{\text {target }}=8$. For the other temperatures $N_{\tau}^{\text {target }}=24,20,12$, this strategy is not immediately applicable. One option is to fold the vacuum data around $m \cdot N_{\tau}^{\mathrm{target}}$, following the recipe of Eq. (6), until the maximum of possible foliations is reached. In this case we assume that the large distance foliations of the vacuum correlator in Eq. (6) provide a negligible contribution to the overall result. An alternative way is to first use a cosh-fit beyond some distance $\tau^{\text {cut }}$ to extend the vacuum correlator data to all $\tau \in \mathbb{R}$. In the following we set $\tau^{\text {cut }} / a=36$. Checking these two methods on the exactly computable $N_{\tau}^{\text {target }}=16$ ensemble, we observe that both of them agree within the statistical errors of the corresponding thermal data.

\section{VECTOR SPECTRAL FUNCTIONS IN TWO-FLAVOR QCD}

With precision data available, we note that two approaches have been widely adopted to reconstruct spectral functions from lattice correlators. The first is the maximum entropy method (MEM) [13, 47 49]. Here Bayes' theorem is invoked to determine the most probable spectral function given the data on the one hand and a so-called default model on the other. Standard algorithms maximize an entropy term to determine the coefficients of a set of transformed basis functions $\mathcal{L}(B(k))$ to obtain an approximation of the spectral function based on the correlator 
data. Since the inverse transformation of the basis functions is known, one thereby obtains also an estimate of the spectral function. The main caveat in this method is the dependence of the results on the default model and, to some extent, on the basis of functions in which the spectral function is expanded. Alternatively, instead of fixing the basis functions and determining their weights, one can define an ansatz $F\left(a_{k}, \omega\right)$, with parameters $a_{k}$, for the spectral function [16, 20, 50]. Naturally, the ansatz introduces a model-dependence and has to be justified. If the fit is successful, one obtains, as in the case of the MEM, a spectral function which describes the lattice data within its statistical uncertainty.

In addition to updating our recent analysis using the fit ansatz approach, we also apply the Backus-Gilbert method [11]. The idea of the method is to determine a local average of the spectral function around a given value of $\omega$ without parametrizing it in any particular way 6 . The weighting factor of the average is called the resolution function.

\section{A. Fit ansatz for the spectral function}

In the first step of this analysis, we reconstruct the vector meson thermal spectral functions using the ansatz approach. Specifically we determine the vacuum and thermal spectral functions from simultaneously fitting all available correlator data $G(\tau, T)$ and enforcing the exact sum rule of Eq. (13).

\section{Combined fit for the vacuum and thermal spectral functions}

To motivate a sensible ansatz for the spectral function on our ' $T=0$ ' ensemble, we first note that the transport contribution is absent in the vacuum. Given the ensemble parameters, we describe the $\rho$ meson contribution by a $\delta$-function. The large frequency behavior is parametrized by a sharp threshold to the perturbative behavior,

$$
\frac{\rho_{V}(\omega, T \simeq 0)}{2 \pi}=a_{V} \delta\left(\omega-m_{V}\right)+\frac{3 \kappa_{0}}{4 \pi^{2}} \Theta\left(\omega-\Omega_{0}\right) \omega^{2} \tanh \left(\frac{\omega \beta_{0}}{4}\right), \quad(\omega \geq 0) .
$$

Based on similar considerations, we define a set of thermal spectral function models for $(\omega \geq 0)$.

As initial setup, we propose that the thermal spectral function consists of a bound state $\delta$-peak, a continuum threshold, a simple $\delta$-peak for the transport and an OPE inspired term $\left(\sim 1 / \omega^{2}\right)$, which is denoted (Mod. 1) in the following. The ansatz reads:

$$
\frac{\rho_{\text {Mod. } 1}(\omega, T)}{2 \pi}=A_{\mathrm{T}} \omega \delta(\omega)+a_{\mathrm{T}} \delta\left(\omega-m_{\mathrm{T}}\right)+\frac{3 \kappa_{0}}{4 \pi^{2}} \Theta\left(\omega-\Omega_{\mathrm{T}}\right) \omega^{2} \tanh \left(\frac{\omega \beta_{\mathrm{T}}}{4}\right)+\frac{3 \kappa_{\mathrm{O}}}{4 \pi^{2}} \Theta\left(\omega-\Omega_{\mathrm{O}}\right) \frac{1}{\omega^{2}} .
$$

To permit statements on the electrical conductivity, we further introduce a Breit-Wigner type transport peak, while keeping the $\delta$-function for the $\rho$ meson. In addition to introducing a finite width for the transport region, we allow that $\kappa_{0}$ splits into two components below the vacuum threshold at $\Omega_{0}$ :

$$
\kappa_{0} \rightarrow \tilde{\kappa}_{0}\left(\kappa_{0}, \kappa_{1}, \Omega_{0}, \eta\right)=\left[\kappa_{0}+\kappa_{1}\left(1-\tanh \left(\frac{\omega}{\Omega_{0} \cdot \eta}\right)^{2}\right)\right] .
$$

Our second and main model is given by (Mod. 2):

$$
\frac{\rho_{\text {Mod.2 }}(\omega, T)}{2 \pi}=\frac{\omega A_{\mathrm{T}} \Gamma_{\mathrm{T}}}{\pi\left(\Gamma_{\mathrm{T}}^{2}+\omega^{2}\right)}+a_{\mathrm{T}} \delta\left(\omega-m_{\mathrm{T}}\right)+\frac{3 \tilde{\kappa}_{0}}{4 \pi^{2}} \Theta\left(\omega-\Omega_{\mathrm{T}}\right) \omega^{2} \tanh \left(\frac{\omega \beta_{\mathrm{T}}}{4}\right)+\frac{3 \kappa_{\mathrm{O}}}{4 \pi^{2}} \Theta\left(\omega-\Omega_{\mathrm{O}}\right) \frac{1}{\omega^{2}} .
$$

\footnotetext{
${ }^{6}$ We note that an alternative model independent reconstruction procedure based on Cuniberti's method was discussed in [51, 52].
} 


\begin{tabular}{lcccccccc}
\hline \hline Ansatz & $N_{\tau} T / T_{c}$ & $a_{T}$ & $m_{T}$ & $\kappa_{1}$ & $\Omega_{T}$ & $\kappa_{\mathrm{O}}$ & $\Omega_{\mathrm{O}}$ \\
\hline (Mod. 1) & 24 & 0.80 & free $m_{V}$ & - & $\Omega_{0}$ & free & $\Omega_{0}$ \\
& 20 & 1.00 & free $m_{V}$ & - & 0 & free & $\Omega_{0}$ \\
& 16 & 1.25 & free $m_{V}$ & - & 0 & free & $\Omega_{0}$ \\
& 12 & 1.67 & free $m_{V}$ & - & 0 & free & $\Omega_{0}$ \\
\hline (Mod. 2a) & 24 & 0.80 & free $m_{V}$ & 0 & $\Omega_{0}$ & free & $\Omega_{0}$ \\
& 20 & 1.00 & free $m_{V}$ & 0 & $\Omega_{0}$ & free & $\Omega_{0}$ \\
& 16 & 1.25 & 0 & - & free & 0 & 0 & - \\
& 12 & 1.67 & 0 & - & free & 0 & 0 & - \\
\hline (Mod. 2b) & 24 & 0.80 & free $m_{V}$ & 0 & $\Omega_{0}$ & free & $\Omega_{0}$ \\
& 20 & 1.00 & free $m_{V}$ & free & 0 & 0 & - \\
& 16 & 1.25 & free $m_{V}$ & free & 0 & 0 & - \\
& 12 & 1.67 & free $m_{V}$ & free & 0 & 0 & - \\
\hline (Mod. 2c) & 24 & 0.80 & free $m_{V}$ & 0 & $\Omega_{0}$ & free & $\Omega_{0}$ \\
& 20 & 1.00 & free $m_{V}$ & free & 0 & 0 & - \\
& 16 & 1.25 & 0 & - & free & 0 & 0 & - \\
& 12 & 1.67 & 0 & - & free & 0 & 0 & - \\
\hline (Mod. 2d) & 24 & 0.80 & free $m_{V}$ & 0 & $\Omega_{0}$ & free & $\Omega_{0}$ \\
& 20 & 1.00 & 0 & - & free & free & 0 & - \\
& 16 & 1.25 & 0 & - & free & 0 & 0 & - \\
& 12 & 1.67 & 0 & - & free & 0 & 0 & - \\
\hline \hline
\end{tabular}

TABLE II. Parameter setup for the ansätze discussed in the text. The unlisted parameter $\Gamma_{T}$ is always free, while $\kappa_{0}$ is a parameter which is shared for all temperatures. The parameter $A_{T}$ is eliminated by the sum rule. Whenever the table contains a "-" the associated parameter does not appear. When the entry equals one of the zero-temperature parameters, it is a shared parameter. Note, that the parameter $a_{T}$ is always constrained to $a_{T}>0$.

As next ingredient we incorporate the sum rule of Eq. (13) and find e.g.

$$
A_{\mathrm{T}}=2\left(\frac{a_{0}}{m_{0}}-\frac{a_{\mathrm{T}}}{m_{\mathrm{T}}}-\frac{3 \kappa_{\mathrm{O}}}{8 \pi^{2} \Omega_{\mathrm{O}}^{2}}-\frac{3 \kappa_{0}}{4 \pi^{2}} \int_{0}^{\infty} d \omega \omega\left[\Theta\left(\omega-\Omega_{\mathrm{T}}\right) \tanh \left(\frac{\omega \beta_{\mathrm{T}}}{4}\right)-\Theta\left(\omega-\Omega_{0}\right) \tanh \left(\frac{\omega \beta_{0}}{4}\right)\right]\right),
$$

for the first model (Mod. 1). An analogous expression for the second model is readily derived. The parameter $A_{\mathrm{T}}$ is eliminated from the thermal fit functions by enforcing the sum rule in a combined fit with the vacuum correlator. In the following we use the vacuum and thermal ansätze to fit the data of all ensembles simultaneously. In addition to eliminating $A_{\mathrm{T}}$ via Eq. (40), we enforce the following conditions: The parameters $\kappa_{0}$ as well as $m_{\mathrm{V}}=m_{\mathrm{T}}$ are shared parameters for all ensembles. Without these constraints the fits lead to considerably less well determined parameters. The threshold parameters $\Omega_{0}$ and $\Omega_{T}$ are shared for the $N_{\tau}=128,24$ ensembles, while $\Omega_{T}$ is set to zero for $N_{\tau}=16$ and 12. The free parameter $\Omega_{T}$ is exclusive to the $N_{\tau}=20$ ensemble and is set according Tab. II] The OPE inspired parameter $\Omega_{\mathrm{O}}$ is set to the vacuum value $\Omega_{0}$.

We consider four variants of the second model, as listed in Tab. II. In the vicinity of the critical temperature, it is difficult to provide an argument for a vacuum-like or a thermal-like structure of the spectral function. By testing variants and determining the stable features we consequently obtain a more systematically reliable result. The first variant, (Mod. 2a), enforces a vacuum structure with a continuous threshold located at $\Omega_{0}$. The final variant, (Mod. 2d), on the other hand leaves the threshold free and assumes a largely thermal structure. Both variants exclude 

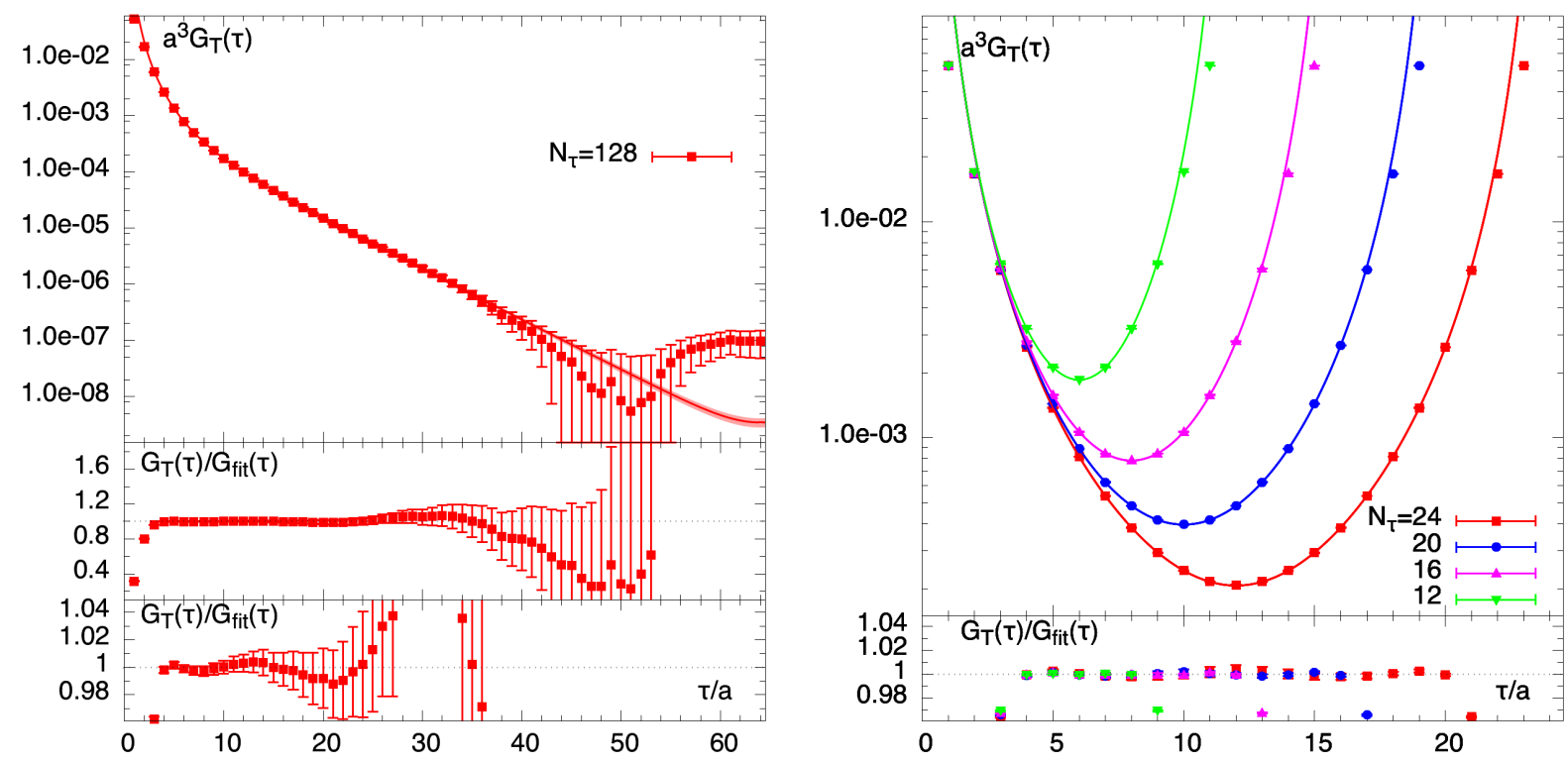

FIG. 1. Left: (Top) The vacuum $\left(N_{\tau}=128\right)$ vector correlation function. The red lines denote the results computed by reconstructing the spectral functions with (Mod. 2c) from the lattice data. The middle panel shows the ratio of the data to the reconstructed result, the bottom panel shows a zoom of this ratio. We observe the lattice data is reproduced with a precision better than $2 \%$ for the distance region $\tau / a \lesssim 20$. Right: (Top) The thermal vector correlators at $T / T_{c}=0.8,1.0,1.25$ and 1.67, i.e. $N_{\tau}=24,20,16$ and 12 . The lines denote the results of the fits based on parametrizing the spectral functions (Mod. 2c). The bottom panel shows the ratio of the data to the fitted correlators.

thermal bound state peaks in the high temperature phase. The second and third variants, (Mod. 2b) and (Mod. 2c), are closely related. Both set the threshold at zero, while allowing a thermal modification via $\kappa_{1}$, but also include a bound state peak for the $N_{\tau}=20$ ensemble. The difference is that (Mod. 2b) allows for bound state peaks in the high temperature phase by setting $a_{T}>0.0$. This model has the most freedom to interpolate between a thermal-like and vacuum-like structure. At the same time, it results in the largest uncertainties on the parameters. Between the tight constraints of (Mod. 2a) or (Mod. 2d) and the relative freedom of (Mod. 2b), the remaining model (Mod. 2c) represents a trade off. Consequently, if not stated otherwise, all results shown in the following are obtained from model (Mod. 2c).

Throughout, the fit range chosen is $\tau / a \in[4: 48]$ for the vacuum and $\tau / a \in\left[4: N_{\tau} / 2\right]$ for the thermal ensembles. We tested additional models, for example also including a Breit-Wigner type peak for the $\rho$ meson. This class of models however lead to badly constrained parameters and a poor description of the data.

\section{Error estimation and results for the correlators}

The combined fit, as outlined above, is carried out on a large number of bootstrap samples, each consisting of the average of $N=1000$ randomly selected data sets, and uses a 'frozen' statistical error obtained from the fully sampled results. The parameters in the fit ansätze are correlated, especially for the bound state and transport regions. To give an error estimate for the parameters we use the method of quantiles as a consequence. As such, the central value parameters are computed on the central value samples of the ensembles. Then we choose the parameter sets corresponding to the central $68 \%$ of the distribution of all solutions as our final results, i.e. we assign 

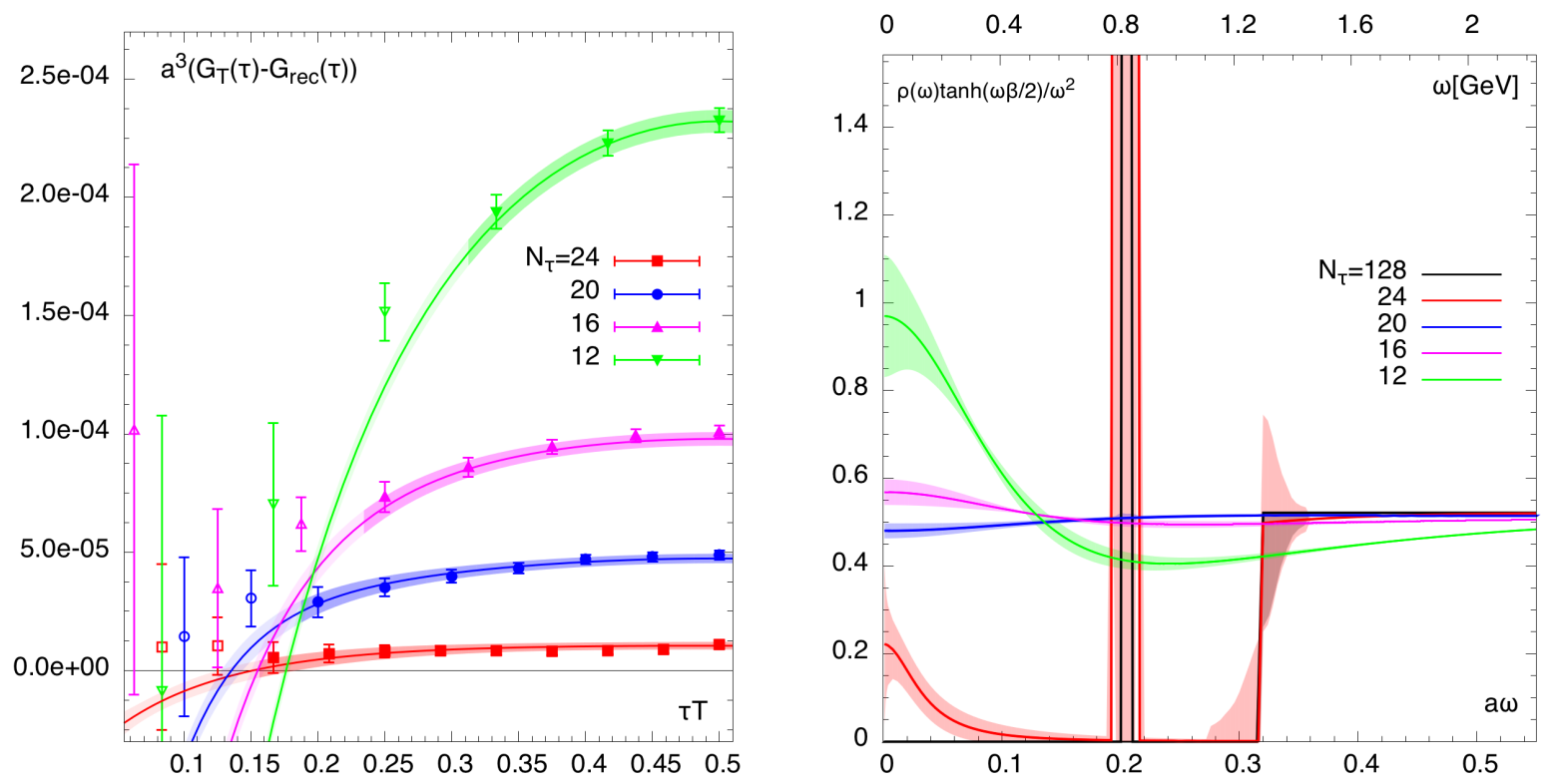

FIG. 2. Left: The difference of thermal and reconstructed correlators together with the results computed by reconstructing the spectral functions from the lattice data. The thermal vector correlators cover the temperature range $T / T_{c}=0.8,1.0,1.25$ and 1.67, i.e. $N_{\tau}=24,20,16$ and 12 , where $T_{c} \simeq 203 \mathrm{MeV}$ and $M_{\pi} \simeq 270 \mathrm{MeV}$. The darker shaded region denotes the fit window used in the reconstruction (Mod. 2c).

Right: The reconstructed spectral functions, rescaled by $\tanh (\omega / 2 T) / \omega^{2}$, in the vacuum (black) and thermal (colors) scenarios from (Mod. 2c).

asymmetric error bars corresponding to one standard deviation. For all derived quantities, such as the correlators recalculated from the spectral functions, we show the standard bootstrapped results in the following. Note, the quoted values for $\chi^{2} /$ d.o.f are 'uncorrelated' values and are collected in Tab. III.

The vacuum correlator is shown in Fig. 1 (left panel). In the top panel of the figure we show the correlator data and the corresponding fit. The middle panel shows the ratio of the data to the reconstructed correlators, the bottom panel shows a zoom of this ratio. We find that our model describes the data accurately within $2 \%$ for distances up to $\tau / a \simeq 20$, i.e. $\simeq 1 \mathrm{fm}$. The corresponding spectral function is displayed in Fig. 2 (right panel).

Likewise, the resulting thermal correlators are shown in Fig. 1 (right panel). In the top panel of this figure we show the correlator data and the corresponding fit, while in the bottom panel we show the ratio of the data and the fit. We observe the fitted model describes the lattice data better than $1 \%$ within the fit range. The thermal spectral functions are also shown in Fig. 2 (right panel).

\section{Discussion of fit results}

We show the results from fitting model (Mod. 2c) to $G(\tau, T)-G_{\text {rec }}(\tau, 0)$ in the left panel of Fig. 2. Here, the shaded bands denote the results from the spectral function reconstruction using the ansatz approach. The data used in the fits is shown in a darker shade and by filled symbols, while the lighter shaded regions and open symbols denote the region outside of the fit windows. For all temperatures we observe good agreement between the data and the results from the fitted spectral functions. 

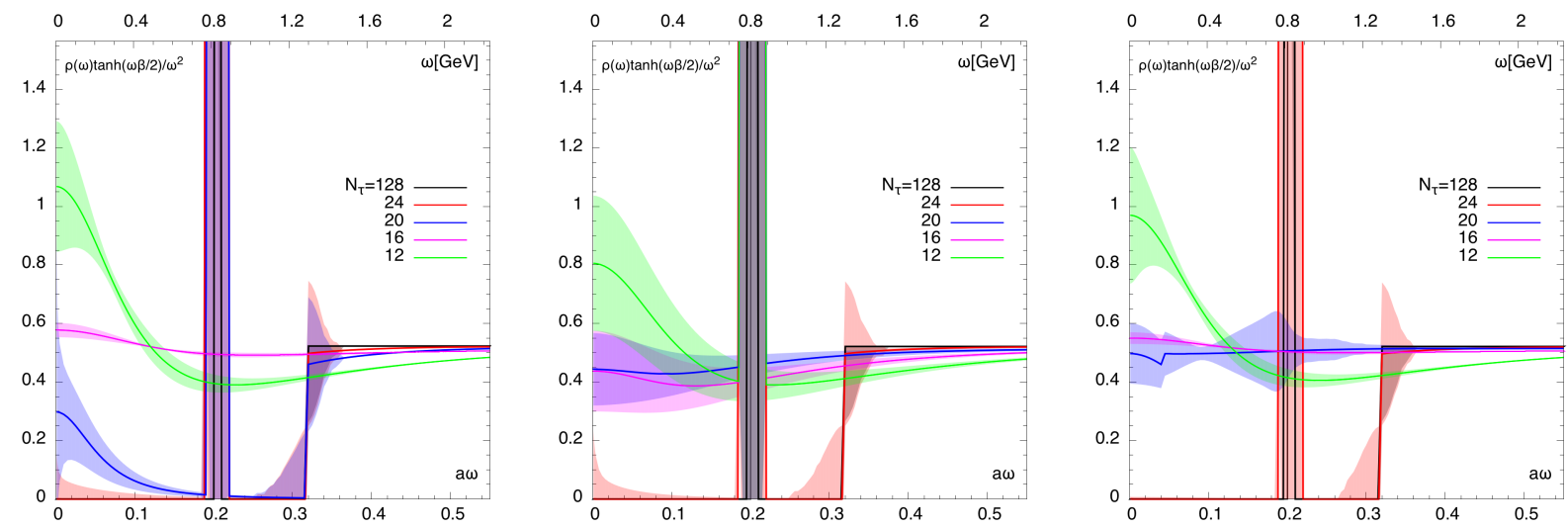

FIG. 3. The reconstructed spectral functions in the vacuum (black) and thermal (colors) scenarios from (Mod. 2a) in the left panel, (Mod. 2b) in the middle and from (Mod. 2d) in the right panel.

In the right panel of Fig. 2 and in Fig. 3 we show the reconstructed spectral functions for (Mod. $2 \mathrm{a}-\mathrm{d})$, rescaled by $\rho(\omega) \rightarrow \rho(\omega) \tanh (\omega \beta / 2) / \omega^{2}$. This rescaling renders the displayed functions finite both at low and large frequencies.

Focusing on low frequencies, i.e. the transport region of the spectral functions in Fig. 2 (right) and Fig. 3, we observe a significant contribution of spectral weight in the transport region for all tested variants above $T \geq T_{c}$. The intercepts of the spectral functions for the $N_{\tau}=20$ ensemble overlap well with each other, where the most vacuum-like variant (Mod. 2b) in Fig. 3(left) appears to be poorly constrained in this region, due to its large uncertainties. In the confined phase $\left(N_{\tau}=24\right)$, we observe a tendency for the suppression of the transport peak and a small change of the bound state peak compared to the vacuum case. Below $\omega=1 \mathrm{GeV}$, we observe a "filling-up" of spectral weight, leading to almost flat spectral functions for $N_{\tau}=20$ and $N_{\tau}=16$. The almost constant behavior of the function $\rho(\omega) \tanh (\omega \beta / 2) / \omega^{2}$ is reminiscent of the spectral function of the R-charge current in the strongly coupled $\mathcal{N}=4$ super Yang-Mills [53; such a spectral function describes a medium with no quasiparticles. At the highest temperature $\left(N_{\tau}=12\right.$ ensemble) we see an excess of spectral weight around the origin as compared to the flat behavior, suggesting that a transport peak is beginning to emerge. At even higher temperatures, the transport peak is expected to become gradually narrower in units of $\omega / T$.

The parameters determining the spectral functions are given in Tab. III, whereby the upper and lower numbers indicate the errors obtained using the quantile method. The amplitudes of the bound state peaks between the vacuum and $N_{\tau}=24$ ensemble are very close to each other, thereby indicating no clear sign of thermal modification. For (Mod. 1) and (Mod. 2a), where a bound state contribution is explicitly included, we observe an increase of the peak amplitude at $T=T_{c}$, i.e. for the $N_{\tau}=20$ ensemble. On the other hand, models based on a more thermal behavior show a strong suppression of the peak amplitude. The spectral weight lost around the $\rho$ mass is seemingly compensated by a modification of $\kappa_{0}$ via $\kappa_{1}$. The width of the transport peaks $\Gamma_{\mathrm{T}}$ are consistent in the last two models (Mod. 2c and Mod. 2d) in the deconfined phase. In addition we observe a trend from broader to narrower widths as the temperature increases. At $T_{c}$, the 'thermal' models (2b, 2c, 2d) agree within errors, while the 'vacuum' model (2a) exhibits larger uncertainties. The parameters in the confined phase show large uncertainties for all models.

The height parameters of the transport peak $A_{\mathrm{T}}$, derived using the sum rule, are listed in Tab. IV. The values using (Mod. 1) are consistently lower in the deconfined phase compared to the variants of (Mod. 2). At the same time they are compatible in the confined phase and at $T_{c}$ when a vacuum-like variant is used. This indicates a very narrow and suppressed transport contribution 


\begin{tabular}{llllll}
\hline \hline Par. [latt. units] & Mod. 1) & (Mod. 2a) & (Mod. 2b) & (Mod. 2c) & (Mod. 2d) \\
\hline$m_{V}$ & $0.205_{5}^{5}$ & $0.205_{5}^{4}$ & $0.205_{6}^{5}$ & $0.206_{4}^{3}$ & $0.205_{6}^{5}$ \\
\hline$a_{V}$ & $0.00085_{9}^{8}$ & $0.00085_{8}^{7}$ & $0.00084_{10}^{8}$ & $0.00085_{6}^{5}$ & $0.00085_{9}^{8}$ \\
$a_{T, N_{\tau}=24}$ & $0.00086_{8}^{8}$ & $0.00086_{10}^{7}$ & $0.00086_{11}^{9}$ & $0.00086_{7}^{6}$ & $0.00086_{10}^{9}$ \\
$a_{T, N_{\tau}=20}$ & $0.00095_{9}^{8}$ & $0.00093_{9}^{8}$ & $0.00011_{5}^{4}$ & $\left.2.8 e^{-9}\right|_{2.5 e^{-9}} ^{1.2 e^{-6}}-$ \\
$a_{T, N_{\tau}=16}$ & $0.00054_{5}^{5}$ & - & $0.00024_{4}^{8}$ & - & - \\
$a_{T, N_{\tau}=12}$ & $0.00048_{11}^{12}$ & - & $0.00014_{7}^{9}$ & - & - \\
\hline$\Gamma_{T, N_{\tau}=24}$ & - & $\left.1.0 e^{-7}\right|_{1.0 e^{-7}} ^{5.0}$ & $\left.1.0 e^{-7}\right|_{1.0 e^{-7}} ^{5.0}$ & $0.03_{3}^{19}$ & $\left.1.0 e^{-7}\right|_{1.0 e^{-7}} ^{3.1 e^{-7}}$ \\
$\Gamma_{T, N_{\tau}=20}$ & - & $0.062_{62}^{19}$ & $0.174_{44}^{170}$ & $0.269_{18}^{17}$ & $0.243_{20}^{20}$ \\
$\Gamma_{T, N_{\tau}=16}$ & - & $0.245_{25}^{52}$ & $0.168_{16}^{72}$ & $0.259_{33}^{24}$ & $0.284_{27}^{25}$ \\
$\Gamma_{T, N_{\tau}=12}$ & - & $0.109_{24}^{23}$ & $0.136_{17}^{95}$ & $0.126_{16}^{11}$ & $0.126_{24}^{26}$ \\
\hline$\kappa_{\mathrm{O}, \mathrm{N}_{\tau}=24}$ & $-0.00014_{25}^{22}-0.00014_{27}^{22}$ & $-0.00015_{3}^{7}$ & $-0.00014_{19}^{28}$ & $-0.00017_{24}^{23}$ \\
$\kappa_{\mathrm{O}, \mathrm{N}_{\tau}=20}$ & $-0.00135_{21}^{20}-0.00155_{29}^{34}$ & - & - & - \\
$\kappa_{\mathrm{O}, \mathrm{N}_{\tau}=16}$ & $-0.00281_{37}^{37}$ & - & - & - & - \\
$\kappa_{\mathrm{O}, \mathrm{N}_{\tau}=12}$ & $-0.00134_{45}^{50}-$ & - & - & - \\
\hline$\kappa_{0}$ & $1.092_{3}^{2}$ & $1.093_{3}^{2}$ & $1.092_{4}^{3}$ & $1.092_{2}^{2}$ & $1.091_{3}^{3}$ \\
$\kappa_{1, N_{\tau}=20}$ & - & - & $-0.058_{38}^{13}$ & $-0.071_{4}^{4}$ & $-0.060_{11}^{25}$ \\
$\kappa_{1, N_{\tau}=16}$ & - & $-0.089_{26}^{9}$ & $-0.072_{29}^{17}$ & $-0.094_{8}^{5}$ & $-0.100_{13}^{10}$ \\
$\kappa_{1, N_{\tau}=12}$ & - & $-0.045_{11}^{11}$ & $-0.081_{43}^{7}$ & $-0.054_{8}^{12}$ & $-0.051_{11}^{11}$ \\
\hline$\Omega_{0}$ & $0.319_{16}^{13}$ & $0.320_{15}^{11}$ & $0.317_{19}^{14}$ & $0.319_{11}^{9}$ & $0.318_{18}^{14}$ \\
$\Omega_{T, N_{\tau}=20}$ & - & - & - & - & $0.045_{45}^{180}$ \\
$\eta$ & - & $2.30_{12}^{17}$ & $2.01_{13}^{32}$ & $2.30_{9}^{14}$ & $2.36_{17}^{21}$ \\
\hline$\chi^{2} / d . o . f$. & 0.56 & 0.60 & 0.54 & 0.54 & 0.51 \\
\hline \hline
\end{tabular}

TABLE III. The parameters in lattice units obtained by fitting the lattice vector meson correlators simultaneously and enforcing the sum rule. The upper and lower errors in the other columns indicate the top and bottom margins from the generally asymmetric error determined by the quantile method. As noted in the text the final results quoted originate from (Mod. 2c). We tested additional model setups and show only those explored fit ansätze that lead to an uncorrelated $\chi^{2} /$ d.o.f $\leq 1.0$.

\begin{tabular}{llllll}
\hline \hline $\mathrm{A}\left[a^{2}\right]$ & (Mod. 1) & (Mod. 2a) & (Mod. 2b) & (Mod. 2c) & (Mod. 2d) \\
\hline$A_{N_{\tau}=24}$ & $0.00023_{7}^{7}$ & $0.00023_{11}^{14}$ & $0.00022_{42}^{20}$ & $0.00032_{22}^{34}$ & $0.00021_{10}^{10}$ \\
$A_{N_{\tau}=20}$ & $0.00062_{12}^{12}$ & $0.00091_{22}^{23}$ & $0.00386_{77}^{285}$ & $0.00646_{28}^{31}$ & $0.00604_{277}^{80}$ \\
$A_{N_{\tau}=16}$ & $0.00095_{37}^{32}$ & $0.00883_{58}^{129}$ & $0.00460_{100}^{211}$ & $0.00918_{66}^{57}$ & $0.00977_{66}^{59}$ \\
$A_{N_{\tau}=12}$ & $0.00376_{87}^{76}$ & $0.00970_{66}^{66}$ & $0.00913_{193}^{276}$ & $0.01019_{50}^{33}$ & $0.01019_{68}^{70}$ \\
\hline \hline
\end{tabular}

TABLE IV. The resulting height parameters of the transport contribution $A_{\mathrm{T}}\left[a^{2}\right]$ in lattice units from combined fitting the lattice vector meson correlators and enforcing the sum rule. As before, the upper and lower errors in the other columns indicate the top and bottom margins from the generally asymmetric error determined by the quantile method.

at $N_{\tau}=24$. For all variants of (Mod. 2) the values of $A_{\mathrm{T}}$ show consistent trends and agree at a qualitative level. As such we observe an increase of $A_{\mathrm{T}}$ with temperature for all models tested.

Taken together, the suppression of a bound state peak in favor of a broad spectral distribution 
in (Mod. 2c) and the consistency between the results of (Mod. 2b)-(Mod. 2d) hint at a rapid dissociation of the narrow bound state as the system crosses over into the deconfined phase. At the same time we see large changes in the electrical conductivity, mirrored by $A_{\mathrm{T}}$ at $T=T_{c}$, where the width of the transport peak acquires a large value that then decreases with temperature.

Although our results are not yet decisive enough to present a definite, ab initio and precision determination of the dissociation of the $\rho$ meson, this is, to our knowledge, the first lattice calculation resolving the spectral weight of a light hadron as it crosses the deconfinement phase transition.

\section{B. The Backus-Gilbert method}

Up to this point the results depend on the chosen model for the true spectral function. Although we believe that our assumptions are plausible, we present in the following a method to constrain the spectral function locally in the variable $\omega$ using only the information contained in the lattice data. This can be achieved by means of the Backus-Gilbert method (BGM) [21]. The method represents a paradigm change from the usual goal of reconstructing the entire spectral function from the lattice correlation function data. We applied this method to the study of pion dispersion relations in [11] and now extend it to the vector spectral functions.

First, define a rescaled kernel,

$$
K(\tau, \omega)=f(\omega / T) \frac{\cosh [\omega(\beta / 2-\tau)]}{\sinh [\omega \beta / 2]},
$$

where $f(x) \stackrel{x \rightarrow 0}{\propto} x$ and $f(x)>0$ for $x>0$. Appropriate choices for $f(x)$ will be discussed later. Now consider a 'resolution function' $\delta\left(\omega, \omega^{\prime}\right)$, such that the 'filtered spectral function'

$$
\widehat{\rho}(\omega) \equiv f(\omega / T) \int_{0}^{\infty} d \omega^{\prime} \delta\left(\omega, \omega^{\prime}\right) \frac{\rho\left(\omega^{\prime}, T\right)}{f\left(\omega^{\prime} / T\right)}
$$

provides an averaged value (in a sense made more precise below) of $\rho$ around frequency $\omega$. For a given $\omega$, the goal is for $\delta\left(\omega, \omega^{\prime}\right)$, viewed as a function of $\omega^{\prime}$, to be a narrowly concentrated function around $\omega$. Restricting ourselves to methods linear in the lattice data, the filtered spectral function is necessarily given by a linear combination of the form

$$
\widehat{\rho}(\omega)=f(\omega / T) \sum_{i=1}^{n} g_{i}(\omega) G\left(\tau_{i}\right),
$$

where $\tau_{i}$ are the Euclidean times, for which the correlator has been calculated. The coefficients $g_{i}(\omega)$ are chosen to realize the stated goal. Inserting the Euclidean correlator into Eq. (43) in terms of $\rho\left(\omega^{\prime}, T\right)$, we conclude that the resolution function is given by

$$
\delta\left(\omega, \omega^{\prime}\right)=\sum_{i=1}^{n} g_{i}(\omega) K\left(\tau_{i}, \omega^{\prime}\right)
$$

For a given $\omega$, it is thus completely specified by the $n$ coefficients $g_{i}(\omega)$. One possible recipe to construct the $g_{i}(\omega)$ is provided by the method of Backus and Gilbert [21], which minimizes the 'width'

$$
\Gamma_{\omega} \equiv \int_{0}^{\infty} d \omega^{\prime}\left(\omega-\omega^{\prime}\right)^{2} \delta\left(\omega, \omega^{\prime}\right)^{2}
$$


of the resolution function for a normalized area

$$
\int_{0}^{\infty} d \omega^{\prime} \delta\left(\omega, \omega^{\prime}\right)=1
$$

The latter condition implies that, if $\rho\left(\omega^{\prime}, T\right) / f\left(\omega^{\prime} / T\right)$ is independent of $\omega^{\prime}$ for $\left|\omega^{\prime}-\omega\right| \lesssim \Gamma_{\omega}$, the filtered spectral function $\widehat{\rho}(\omega)$ is an estimator for the value of $\rho(\omega, T)$ at the point $\omega$. The solution is (see for instance [54])

$$
g_{i}(\omega)=\frac{\left(W^{-1}\right)_{i j} R_{j}}{R_{k}\left(W^{-1}\right)_{k l} R_{l}}
$$

with

$$
W_{i j}(\omega)=\int_{0}^{\infty} d \omega^{\prime} K\left(\tau_{i}, \omega^{\prime}\right) K\left(\tau_{j}, \omega^{\prime}\right)\left(\omega-\omega^{\prime}\right)^{2} \quad \text { and } \quad R_{i} \equiv \int_{0}^{\infty} d \omega K\left(\tau_{i}, \omega\right)
$$

In practice, the matrix $W$ rapidly becomes ill-conditioned with increasing $n$, the coefficients $g_{i}(\omega)$ become large and alternating in sign, which is a manifestation of the ill-posed nature of the inverse problem. It is necessary to regularize the procedure by replacing $W$ with

$$
W_{i j}^{\text {reg. }}(\omega)=\lambda W_{i j}(\omega)+(1-\lambda) \operatorname{Cov}_{i j}[G], \quad 0 \leq \lambda \leq 1,
$$

where $\operatorname{Cov}[G]$ is the covariance matrix of the lattice correlator $G\left(\tau_{i}, T\right)$. The Backus-Gilbert method can then be thought of as minimizing the width $\Gamma_{\omega}$ under the condition that the statistical error on $\widehat{\rho}(\omega, T)$ assumes a preset value. Increasing $\lambda$ slowly reduces $\Gamma_{\omega}$ at the cost of increasing the statistical errors. The only input into the construction of the resolution function is thus the covariance matrix, the choice of $f(x)$ and the value of the regularization parameter $\lambda$. Independent of the choices made, it is clear that quoting $\widehat{\rho}(\omega, T)$ at $n$ values of $\omega$-typically spaced by separations of order $\Gamma_{\omega}$ - is equivalent information to the original lattice data; one has simply deconvoluted the information as local in $\omega$ as the quality of the lattice data allows. We note that the resolution function has already been used in [55] as a way to provide a rigorous meaning to a linear reconstruction method for the spectral function.

We now discuss the choice of the reweighting function $f(x)$. It is desirable to choose it in such a way, that the reconstructed function, $\rho(\omega, T) / f(\omega / T)$, does not show a global trend. For the vector channel considered here, we note that $\left(\rho_{i i}(\omega, T) \frac{T^{2}}{\omega^{2}} \tanh \frac{\omega}{2 T}\right)$ is expected to be finite in both limits, $\omega \rightarrow 0$ and $\omega \rightarrow \infty$. We therefore choose

$$
f(x)=\frac{x^{2}}{\tanh (x / 2)} .
$$

We will also consider the vacuum-subtracted correlation functions, $G(\tau, T)-G_{\mathrm{rec}}(\tau, 0)$. Since we expect $\Delta \rho(\omega) \stackrel{\omega \rightarrow \infty}{\propto} 1 / \omega^{2}$ from the operator-product expansion, we choose

$$
f^{\Delta}(x)=\tanh (x / 2)
$$

as reweighting function. Various reweighting functions were already introduced in [11, 13, 14, 56, 57, to remove the divergence of the kernel at the origin. Here, the reweighting function plays the additional role of rescaling the spectral function to remove its overall growth at large $\omega$.

Finally, we remark that many other choices than Eq. (45) are possible as a measure of the width of $\delta\left(\omega, \omega^{\prime}\right)$. The most useful choice depends on the channel under consideration as well as what question precisely one is addressing. 

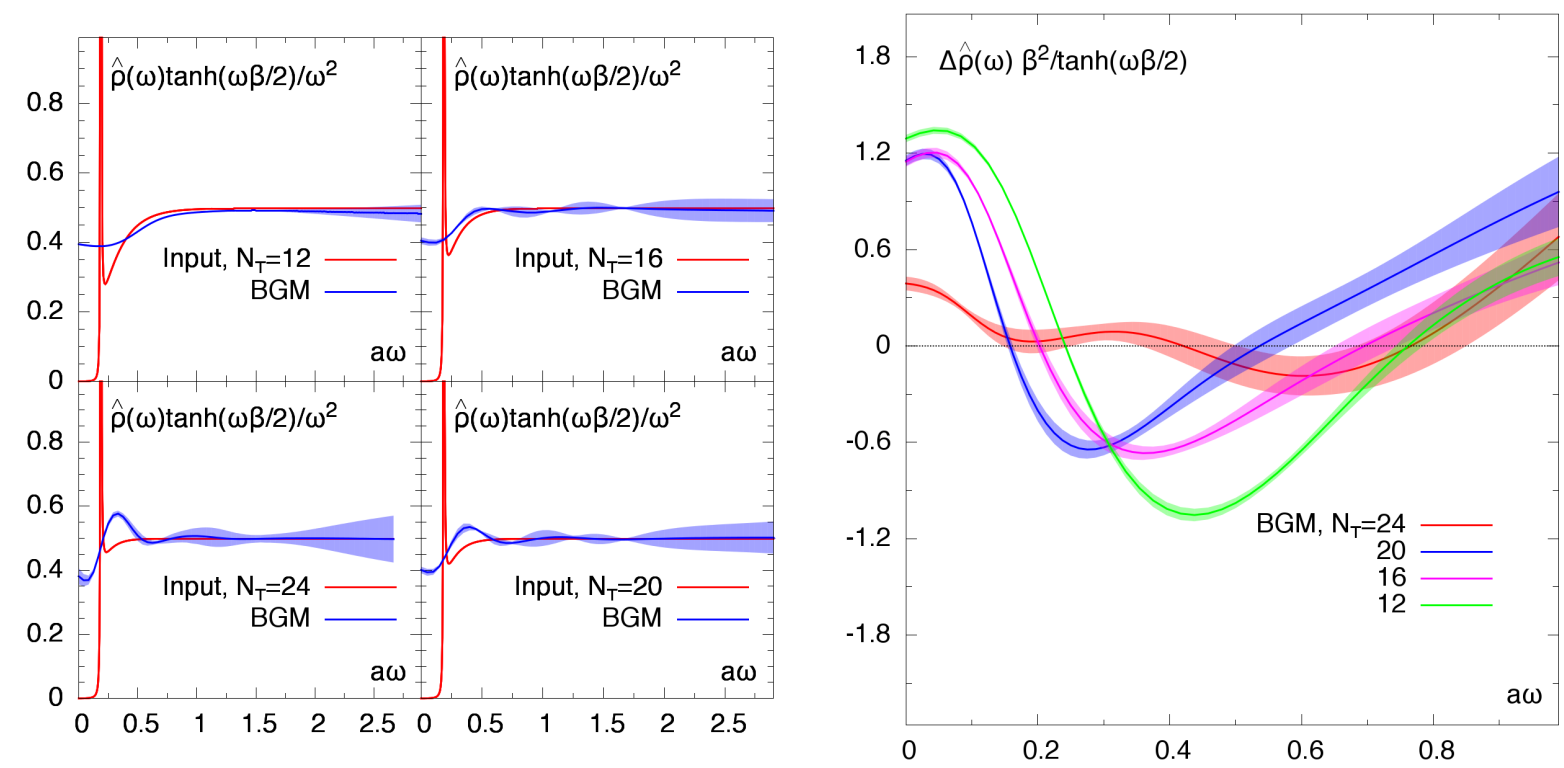

FIG. 4. Left: Mock data test of the Backus-Gilbert method, reconstructing $\widehat{\rho}(\omega)$ from a known spectral function (red) and the covariance matrix supplied by the lattice data. Right: The convoluted spectral functions for the difference $\Delta \widehat{\rho}(\omega)$ from reconstructing $G(\tau, T)-G_{\text {rec }}(\tau, 0)$ using the BGM. The narrower resolution function and favorable cancellation of the large frequency regions enable more details to be reconstructed.

\section{Applying the BGM: tests and results}

We found that a value of the regularization parameter $\lambda=0.002$ was a reasonable choice for all ensembles, although larger values up to $\lambda=0.9$ lead to equally good results for the high temperature lattices $N_{\tau}=12$ and 16. The regularization is crucial for the lower-temperature lattices, when a large number of points enter $W_{i j}(\omega)$.

To illustrate the precision that can be achieved, we show the resolution function $T \delta\left(\omega, \omega^{\prime}\right)$ computed on the $N_{\tau}=24$ ensemble, as a function of $\omega^{\prime} / T$, for the four values $\omega / T=0,4,8$ and 16 in Fig. 5 (left panel). As input we choose the points $\tau_{i}=(3, \ldots, 12)$ in $G(\tau)$. We observe that the width of the resolution function increases with increasing frequency $\omega$, from roughly $\Gamma_{\omega} \sim 5 T$ at $\omega / T=0$ to $\Gamma_{\omega} \sim 15 T$ at $\omega / T=8$. Since the resolution function only depends on the kernel and the precision of the lattice data, this sets limits on the frequency resolution that can be reached in our lattice calculation. The relatively poor resolution is partly due to the rise $\sim \omega^{2}$ of the spectral function of the conserved vector current.

Next we perform a mock data test to assess the relation between $\widehat{\rho}(\omega, T)$ and $\rho(\omega, T)$ in the vector case. To this end we replace the average values of the lattice correlator by pseudodata derived from a known spectral function, which we chose to be the fitted vacuum result, and keep the lattice covariance matrix. As before the points chosen as input to the method are $\tau_{i}=\left(3, \ldots, N_{\tau} / 2\right)$. The resulting filtered spectral functions, $\widehat{\rho}(\omega)$, are shown in Fig. 4 (left panel) for all four available lattice ensembles. With increasing temperature we observe the sharp features of the input mock spectral function to be washed out by the filtering effect of the resolution function.

In the last step, we apply the Backus-Gilbert method to actual lattice data, both for the correlator $G(\tau, T)$ itself and for the difference $G(\tau, T)-G_{\text {rec }}(\tau, 0)$. The results of the latter case are displayed in red in Fig. 4 (right panel), while the former are given in Fig. 5 (left). The narrower resolution function and the cancellation of the large frequency behavior in $\Delta \rho(\omega, T)$ allow more 


\begin{tabular}{lccl}
\hline \hline$T[\mathrm{MeV}]$ & $\chi_{s} / T^{2}$ & $T \delta\left(\omega_{0}=0, \omega=0\right)$ & $\sqrt{\left\langle v^{2}\right\rangle_{\mathrm{eff}}}$ \\
\hline 169 & $0.42718(31)$ & 0.36588 & $0.629(34)$ \\
203 & $0.72120(29)$ & 0.37275 & $0.826(12)$ \\
254 & $0.86771(34)$ & 0.37484 & $0.749(10)$ \\
338 & $0.93949(25)$ & 0.37250 & $0.7661(68)$ \\
\hline \hline
\end{tabular}

TABLE V. The static susceptibility and the effective root-mean-square velocities, obtained on the four thermal lattice ensembles using the BGM filtered thermal spectral functions. The resolution function is given in the third column.

structure to be extracted from the lattice data. See the right panel of Fig. 5 for the corresponding resolution function at $T=169 \mathrm{MeV}$. As the temperature is increased we observe the emergence of a negative dip around the vector meson mass, along with an enhancement in the transport region. This suggests the dissociation of the $\rho$ meson and the formation of a transport peak. This dip forms already at $N_{\tau}=20$, corroborating to the conclusion of section IV A 3, that there is a rapid dissociation of the bound state around $T_{c}$ accompanied by a rapid build-up of the transport contribution.

\section{COMPARISONS WITH MODEL PREDICTIONS}

\section{A. Comparing lattice results to the HRG model}

As discussed in section II, the hadron resonance gas (HRG) model makes definite predictions for the the static susceptibility $\chi_{s}$ and the mean-square-velocity of the hadronic quasiparticles in the low-temperature phase of QCD. Here we compare these predictions to the results obtained from our lattice simulations.

On the lattice, the static susceptibility is computed straightforwardly using Eq. 22). The meansquare velocity is more difficult to estimate, since it must be extracted from the spectral function and assumes the presence of a well-defined transport peak. To construct an estimator for the mean-square velocity, we use the BG filtered spectral function $\widehat{\rho}(\omega, T)$ to write

$$
\left\langle v^{2}\right\rangle_{\mathrm{eff}}=\left.\frac{1}{2 \pi \chi_{s} T \delta(0,0)} \frac{\Delta \widehat{\rho}(\omega, T)}{\tanh (\omega \beta / 2)}\right|_{\omega=0},
$$

which is based on Eq. (25). It relies on the narrowness of the transport peak compared to the width of the resolution function $\delta(0, \omega)$. We focus the following discussion on the model independent results obtained using the BGM and present the static susceptibility, the value of $T \cdot \delta(0,0)$ for the resolution function and the mean-square velocities in Tab. V.

To obtain an HRG prediction at the physical $(u, d)$ quark masses, we would sum up the contributions to $\chi_{s}$ and $\chi_{s}\left\langle v^{2}\right\rangle$ of the mesons and baryons listed in the Particle Data Group [58] up to a mass of about $2 \mathrm{GeV}$. Since the quark masses in our lattice simulations are larger than in nature, we attempt to correct for the bulk of this effect by setting the pion, $\rho$ and nucleon masses to the values found in our lattice ensembles. For the other mesons and baryons, we have added a quark-model inspired shift to the PDG masses equal to $m_{\rho}^{\text {latt }}-m_{\rho}^{\text {phys }}$ and $m_{N}^{\text {latt }}-m_{N}^{\text {phys }}$ respectively. Since we perform two flavor simulations, hadrons with non-vanishing strangeness or charm are not included. We thus obtain the following HRG estimates for the two-flavor theory with a zero-temperature 
pion mass of $\simeq 270 \mathrm{MeV}$,

$$
\text { HRG : } \quad \chi_{s} / T^{2}=\left\{\begin{array}{ll}
0.49 & T=169 \mathrm{MeV} \\
0.93 & T=203 \mathrm{MeV}
\end{array}, \quad \sqrt{\left\langle v^{2}\right\rangle}=\left\{\begin{array}{ll}
0.74 & T=169 \mathrm{MeV} \\
0.70 & T=203 \mathrm{MeV}
\end{array} .\right.\right.
$$

The temperature evolution of the mean-square velocity originates from two competing effects: As the temperature rises, heavier hadrons have a chance to appear in the medium with a small velocity. On the other hand, lighter hadrons become gradually more relativistic. Thus a meansquare velocity which decreases as a function of temperature signals the approach to the Hagedorn regime where the appearance of new hadron species dominates. In our model, $\left\langle v^{2}\right\rangle$ has a maximum around $T=130 \mathrm{MeV}$.

Comparing the static susceptibilities in Eq. (53) and in Tab. V, we find a reasonably good agreement at $T=169 \mathrm{MeV}$, but a clear overestimate of the HRG prediction at $T=203 \mathrm{MeV}$. The HRG value is in fact already close to the Stefan-Boltzmann limit value of $\chi_{s} / T^{2}=1$.

Looking at the mean-square velocities, we start with the ensemble at $T=169 \mathrm{MeV}$. The width of $\delta(0, \omega)$ is approximately $\frac{\pi}{2} T$ in our study. In this regime, the quantity $\left\langle v^{2}\right\rangle_{\text {eff }}$ is a good estimator of the mean-square velocity of quasiparticles, if (a) the transport peak at the origin in $\rho_{i i}(\omega, T)$ exists and is narrow in comparison with the thermal scale $\omega \sim \pi T$ and (b) the next significant contribution to the subtracted spectral function $\Delta \rho(\omega, T)$ lies well beyond the width of $\delta(0, \omega)$. As for (a), kinetic theory estimates ${ }^{7}$ of the width of the transport peak based on a hadronic gas picture clearly indicate that the width is much smaller than $T$. Assuming this to be true at the lowest temperature $T=169 \mathrm{MeV}$, we observe that the lattice estimate of $\sqrt{\left\langle v^{2}\right\rangle} \approx 0.63$ is somewhat smaller than the HRG value 0.74. This observation is interesting, since we found on the same lattice ensemble in our recent study [11 that the pion quasiparticle is lighter than the zero-temperature pion mass and has a less steep dispersion relation, $\omega_{\vec{p}}^{2}=m_{\text {quasip }}^{2}+u^{2} \vec{p}^{2}$ with $u=0.74(1)$ and $m_{\text {quasip }}=223(4) \mathrm{MeV}$. An alternative to the HRG model is then to only include the pion contribution, however taking into account the modified dispersion relation at low momenta,

$$
\begin{aligned}
\chi_{s} & =4 \beta \int_{|\vec{p}|<\Lambda_{p}} \frac{d^{3} \vec{p}}{(2 \pi)^{3}} f^{B}\left(\omega_{\vec{p}}\right)\left(1+f^{B}\left(\omega_{\vec{p}}\right)\right)+4 \beta \int_{|\vec{p}|>\Lambda_{p}} \frac{d^{3} \vec{p}}{(2 \pi)^{3}} f_{\vec{p}}^{B}\left(1+f_{\vec{p}}^{B}\right), \\
\chi_{s}\left\langle v^{2}\right\rangle & =4 \beta \int_{|\vec{p}|<\Lambda_{p}} \frac{d^{3} \vec{p}}{(2 \pi)^{3}} f^{B}\left(\omega_{\vec{p}}\right)\left(1+f^{B}\left(\omega_{\vec{p}}\right)\right) v_{g}(\vec{p})^{2}+4 \beta \int_{|\vec{p}|>\Lambda_{p}} \frac{d^{3} \vec{p}}{(2 \pi)^{3}} f_{\vec{p}}^{B}\left(1+f_{\vec{p}}^{B} \frac{\vec{p}^{2}}{E_{\vec{p}}^{2}},\right.
\end{aligned}
$$

where $f^{B}(E)=\left(e^{\beta E}-1\right)^{-1}$ and $v_{g}(\vec{p})=\frac{d \omega_{\vec{p}}}{d|\vec{p}|}$ is the group velocity of the pion. In this model, the contribution of the other hadrons are at least partly taken into account indirectly, since it is the collisions of the pions among themselves and with other hadrons which give rise to the modified pion dispersion relation. With a choice of $\Lambda_{p}=500 \mathrm{MeV}$, which is about the momentum scale at which the predictions of the thermal chiral effective theory were seen to break down in [11, one obtains $\chi_{s} / T^{2}=0.43$ and $\sqrt{\left\langle v^{2}\right\rangle}=0.68$, in better agreement with the lattice data than the HRG model.

There are however alternative explanations for the difference between the HRG prediction and the lattice result for $\left\langle v^{2}\right\rangle$. One explanation could be that our lattice value for $\left\langle v^{2}\right\rangle$ is somewhat underestimated. Indeed, this could happen if assumption (b) stated in the previous paragraph were imperfectly realized and if the spectral weight of the $\rho$ meson was somewhat lower than at $T=0$. After all we know from the sum rule in Eq. (13) that some negative spectral weight in $\Delta \rho(\omega, T)$ must appear to compensate the transport peak. A further possible explanation that ought to be studied is the influence of the finite volume in our simulation. Roughly, the average number of

\footnotetext{
${ }^{7}$ E.g. $\Gamma=\sigma n v$, with $\sigma$ a typical hadronic cross-section and $n, v$ the density and the velocity of quasiparticles. Also, the thermal width of the pion quasiparticle calculated in chiral perturbation theory [59] is quite small.
} 

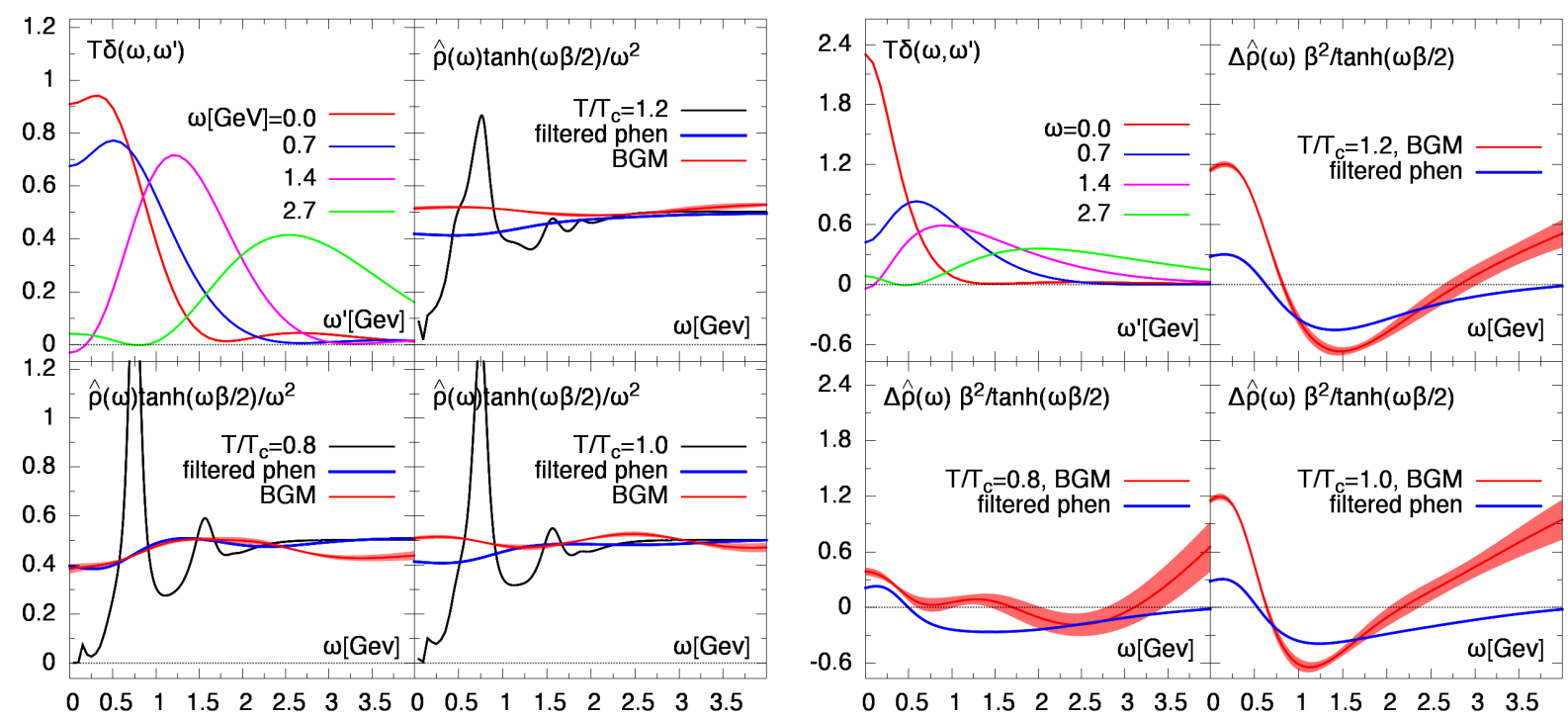

FIG. 5. Left: Comparison of filtered spectral functions $\widehat{\rho}(\omega)$ obtained by convoluting with the resolution functions $\delta\left(\omega, \omega^{\prime}\right)$ determined from the lattice, (red) BGM reconstruction, (blue) spectral functions from phenomenology [22. For comparison, the un-filtered result is displayed in black. The top left panel shows the resolution functions. Right: The same plot for the difference $\Delta \widehat{\rho}(\omega)$. Note, the phenomenological spectral functions do not contain a transport contribution, we therefore do not expect good agreement in the low frequency region at $T>T_{c}$.

hadrons in our $(3 \mathrm{fm})^{3}$ box is of the order of seven, based on the HRG model, which could invalidate a kinetic theory description.

At $T=203 \mathrm{MeV}$, we find using the lattice correlators that the effective rms velocity has risen significantly from its value at $T=169 \mathrm{MeV}$, while the HRG model predicts a slight decrease. The respective values are 0.83 and 0.70 . It is more difficult to explain an overestimate from systematic effects affecting $\sqrt{\left\langle v^{2}\right\rangle_{\text {eff }}}$. Considering the static susceptibility, the rms velocity and our finding in section IV A 3 that the spectral weight of the $\rho$ meson is significantly reduced at a temperature of $200 \mathrm{MeV}$, we conclude that the medium can no longer be thought of as consisting of weakly interacting hadrons flying past each other.

In the high-temperature phase, the data in Table $\mathrm{V}$ shows that the static susceptibility $\chi_{s}$ approaches unity in units of $T^{2}$, as expected. The expected quasiparticles at sufficiently high temperatures are quarks and gluons and we find a relatively low value of the effective rms velocity. A possible explanation is that no well-defined transport peak exists at $T=254 \mathrm{MeV}$ and $T=340 \mathrm{MeV}$. And if the transport peak is present, the width of the resolution function may be too broad to yield an accurate estimate of the rms quasiparticle velocity.

\section{B. Comparison with the thermal spectral functions of Hohler and Rapp}

In 22] the authors used QCD and Weinberg sum rules to study thermal, isovector vector and axial-vector spectral functions across the phase transition. The in-medium condensates, required in this analysis, were estimated using the hadron resonance gas model and lattice QCD data where possible. In the following, we compare these phenomenological spectral functions with those determined from our lattice correlation functions. However, in such a comparison a number of issues have to be considered, e.g. the pion masses are larger than in nature and the calculations 
presented here are not in the continuum limit. In addition, the lattice spectrum in a finite volume consists of a discrete set of delta functions. A direct comparison between spectral functions is therefore not meaningful. On the other hand, comparing Euclidean correlation functions obscures the physical interpretation, which is normally based on the local behavior of the spectral function around a given frequency. Hence the BGM provides an opportunity to compare phenomenological and lattice calculations via the filtered phenomenological spectral function. Indeed, all that is needed for this comparison is the resolution function. A possible recipe to compare $\widehat{\rho}(\omega)$ is to filter the given spectral functions $\rho_{\text {phen }}\left(\omega^{\prime}\right)$ with the resolution functions determined by the lattice data,

$$
\widehat{\rho}_{\text {phen }}(\omega)=\int_{0}^{\infty} d \omega^{\prime} \delta\left(\omega, \omega^{\prime}\right) \rho_{\text {phen }}\left(\omega^{\prime}\right)
$$

To map the phenomenological curves of [22] to our results, we set $T_{c}=155 \mathrm{MeV}$ in QCD with physical $u, d, s$ quark masses [60, 61], entailing the following temperatures $T_{\text {phen }}$ where the phenomenological spectral functions will be evaluated,

$$
\begin{aligned}
& N_{\tau}=24: \quad T / T_{c} \approx 0.8 \Rightarrow T_{\text {phen }}=130 \mathrm{MeV} \\
& N_{\tau}=20: \quad T / T_{c} \approx 1.0 \Rightarrow T_{\text {phen }}=150 \mathrm{MeV}, \\
& N_{\tau}=16: \quad T / T_{c} \approx 1.25 \Rightarrow T_{\text {phen }}=170 \mathrm{MeV}
\end{aligned}
$$

Furthermore we normalize phenomenological curves to the lattice spectral functions by dividing out the charge factor $C_{\mathrm{em}}=5 / 9$. In the left panel of Fig. 5 we show the results for the filtered spectral functions $\widehat{\rho}(\omega)$ and in the right one those for the difference $\Delta \widehat{\rho}(\omega)$. We present our results for the BGM (red), the filtered phenomenological (blue) spectral functions and the unfiltered phenomenological spectral functions (black) in Fig. 5 (left). As a transport contribution is not included in the phenomenological data, we do not expect very good agreement in the low frequency region for temperatures above $T_{c}$. Nevertheless, the qualitative agreement between the phenomenological and reconstructed lattice results is good. Even though a full quantitative study has to be postponed, the presented method allows for a systematic comparison of BGM reconstructed lattice spectral functions and phenomenological results in the future.

\section{ELECTRICAL CONDUCTIVITY ACROSS THE PHASE TRANSITION}

In this section we discuss the impact of our results on our knowledge of the electrical conductivity across the deconfinement phase transition. We recall that the electrical conductivity can be read off from the spectral functions via Eq. (15) from the intercept of $\rho(\omega) / \omega$ at $\omega=0$. Since the BGM gives access only to the filtered spectral functions $\widehat{\rho}(\omega)$, we cannot determine a model independent result for $\sigma_{\text {el }}$ at this time. Nonetheless, we present the results obtained from the ansatz approach in Tab. VI. We quote central values obtained with (Mod. 2c), and use (Mod. 2d) as a way to estimate the systematic error from the choice of ansatz.

Here the errors are determined from the spectral functions for (Mod. 2c) and (Mod. 2d). The first value denotes those from (Mod. 2c), the second gives the lower bounds from (Mod. 2d) relative to the central values of (Mod. 2c) and the third the corresponding upper bound. We observe and increase of the electrical conductivity across the phase transition.

Both the ansatz method and MEM introduce a significant degree of systematic uncertainty, due to the ill-posed nature of the inverse transformation. Consequently cross-checks using multiple approaches are mandatory. In Fig. 6 we collect and compare different determinations of $\sigma_{\mathrm{el}}$ from recent lattice determinations using staggered quenched (grey) [13, Wilson-Clover quenched (red) [15], Wilson-Clover quenched in the continuum limit (blue triangles and magenta bars) [16 18], 


\begin{tabular}{llcl}
\hline \hline$T[\mathrm{MeV}]$ & $T / T_{c}$ & $\sigma_{\mathrm{el}} / C_{\mathrm{em}} T$ & $\Delta \sigma_{\mathrm{el}} / C_{\mathrm{em}} T$ \\
\hline 169 & 0.8 & 0.074 & $(61)(-74)(+0)$ \\
203 & 1.0 & 0.160 & $(6)(-29)(+40)$ \\
254 & 1.25 & 0.189 & $(10)(-13)(+4)$ \\
338 & 1.67 & 0.323 & $(46)(-78)(+78)$ \\
\hline \hline
\end{tabular}

TABLE VI. The electrical conductivity $\sigma_{\text {el }}$ determined via the ansatz approach. The central value (third column) is obtained with (Mod. 2c). The uncertainties, displayed in the fourth column, are determined from the bootstrap samples. The first value is the statistical error of (Mod. 2c). The second and third give the range of values (relative to the central value of the third column) obtained with (Mod. 2d).

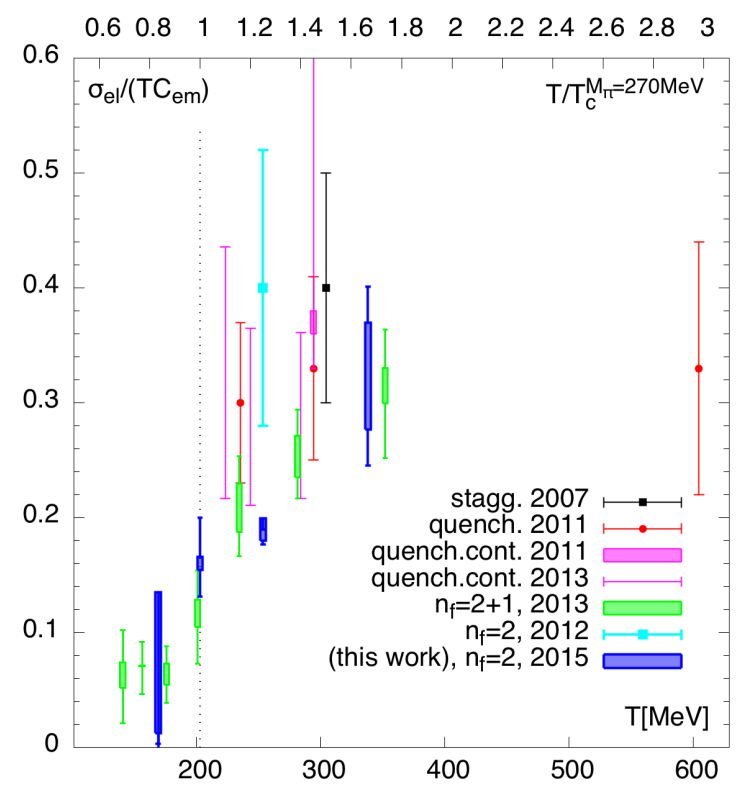

FIG. 6. The electrical conductivity read off from the fitted spectral functions. For our quoted results (blue) the boxes give the errors determined from (Mod. 2c) and the errorbars those from (Mod. 2d). The results from this work are compared to past staggered (grey) [13, quenched (red) [15, quenched in the continuum limit (blue triangles and magenta bars) [16 18, $n_{f}=2+1$ (green whiskerbars) [19], as well as our $n_{f}=2$ [20] studies.

$n_{f}=2+1$ (green whiskerbars) [19], as well as our previous $n_{f}=2$ (blue) Wilson-Clover studies [20. Here the staggered and Wilson-Clover $n_{f}=2+1$ results use MEM for the reconstruction, while the Wilson-Clover quenched and our $n_{f}=2$ studies use the ansatz method. Note, in our previous study [20] we computed the local-local current correlators with a factor 64 lower statistics. Additionally the model did not permit the separation of the contributions for the thermal and vacuum bound states. In the quenched calculations no clear temperature dependence can be seen, while there is a consistent drop in dynamical QCD. This might be due to the different nature of the deconfinement phase transitions in both theories. Comparing especially with the $n_{f}=2+1$ MEM [19] dynamical results, the results are in good agreement. A consistent picture is emerging for the electrical conductivity around the critical temperature $T_{c}$ from lattice simulations. 


\section{SUMMARY AND OUTLOOK}

We have presented the Euclidean correlation functions in the isovector vector channel computed using lattice QCD at temperatures $T / T_{c}=0.8,1.0,1.25$ and 1.67. We have analyzed the correlation functions in terms of the associated spectral functions. The simulations were performed with two flavors of light quarks and included a reference simulation at zero temperature. In this setup, the pseudocritical temperature amounts to $T_{c}=203 \mathrm{MeV}$. With high precision Monte-Carlo data available for all lattice ensembles, we applied a set of sophisticated ansätze and exploited an exact sum rule (Eq. (13)) constraining the vacuum-subtracted spectral function to describe the underlying spectral functions.

Fairly general arguments based on kinetic theory suggest that the $\rho$ meson is largely unaffected by thermal effects as long as the conserved-charge fluctuations and the spectral weight of the transport peak are as small as predicted by the hadron resonance gas model (section IIE). By fitting the ansatz parameters to the lattice data, we find that the spectral weight below the zerotemperature threshold $\omega=2 m_{\pi}$ remains small at $T=0.8 T_{c}$, but becomes significant at $T_{c}$. This thermal effect is accompanied by a reduction of the spectral weight above the threshold, particularly around the vector meson mass $m_{\rho}$. This may be interpreted as evidence of a rapid dissociation of the vector meson as the system crosses into the deconfined phase. At $T=T_{c}$ we find that the hadron resonance gas predictions fail to describe the charge fluctuations (section $\mathrm{VA}$ ). For $T>T_{c}$, the fitted spectral functions do not exhibit any particular excess of spectral weight around the $\rho$ meson mass (Fig. 2).

Using the appropriate Kubo formula, we estimated the electrical conductivity across the deconfinement phase transition (Fig. 6) and compared it with recent lattice QCD calculations. We observe a rapid increase of the electrical conductivity across the deconfinement phase transition in line with that observed in other dynamical calculations. A consistent picture is emerging.

In addition to providing a phenomenologically motivated form of the spectral function which successfully describes the lattice correlation function data, we applied the Backus-Gilbert method to the lattice data. The main advantage of this method is that a 'filtered' spectral function is constructed, which, at any given frequency, is given as a local weighted average of the true spectral function with a known weight function. In this method the spectral function does not have to be expanded in any specific basis of functions and the ill-posed nature of the inverse problem translates into the limited locality in frequency that can be achieved in the relation between the filtered and the actual spectral function. We also point out that the filtered spectral function obtained using the Backus-Gilbert method, does not necessarily provide a description of the lattice data with a small $\chi^{2}$, once integrated with the appropriate kernel in Eq. (3). In that sense, the method is complementary to applying a fit ansatz for the spectral function, or to applying the maximum entropy method.

Having obtained the filtered spectral functions at $T=0.8 T_{c}$, we compared the low-frequency region to the predictions of the hadron resonance gas model (section $\mathrm{VA}$ ) and found agreement at the 10-15\% level. The agreement is somewhat improved if a pion gas is assumed with a modified dispersion relation at low momenta [11. At $T=T_{c}$, as already stated above, the hadron resonance gas model fails. Secondly, we compared the filtered spectral functions to previously published [22] phenomenological spectral functions. Once they are passed through the same filter, they exhibit the same qualitative feature of having excess spectral weight around $\omega=0$ and a depletion at and above the $\rho$ meson mass, as compared to the respective vacuum spectral functions. Quantitatively, we see differences, which could be partly understood by the fact that the zero-temperature pion mass is $m_{\pi} \simeq 270 \mathrm{MeV}$ in our lattice simulations, rather than the physical value of $m_{\pi}=140 \mathrm{MeV}$. We think that comparing the filtered spectral functions is a good way of testing phenomenological models, since it does not introduce model-dependence on the lattice side and is on the other hand 
as local in frequency as the quality of the lattice data allows.

Looking to the future, it would be highly desirable to perform a continuum extrapolation of the lattice data before undertaking spectral analyses. Second, achieving high resolution in frequency space requires very high accuracy. We estimate that cleanly separating the transport contribution from the $\rho$ meson spectral weight at $T=0.8 T_{c}$ in the Backus-Gilbert method would require reducing the error bars by a factor four. Finally, the finite-size effects on the filtered spectral function should be studied in more detail (qualitatively, the effects were discussed in [4]).

\section{ACKNOWLEDGMENTS}

We thank Ralf Rapp and Paul M. Hohler for discussions and for providing their spectral functions. We are grateful for the access to the zero-temperature ensemble used here, made available to us through CLS. We acknowledge the use of computing time for the generation of the gauge configurations on the JUGENE and JUQUEEN computers of the Gauss Centre for Supercomputing located at Forschungszentrum Jülich, Germany. Part of the configurations and all correlation functions were computed on the dedicated QCD platforms "Wilson" at the Institute for Nuclear Physics, University of Mainz, and "Clover" at the Helmholtz-Institut Mainz. This work was supported by the Center for Computational Sciences in Mainz as part of the Rhineland-Palatinate Research Initiative and by the DFG grant ME 3622/2-1 Static and dynamic properties of QCD at finite temperature.

\section{Appendix A: Correlation function data}

Below we list the results of $G_{i i}(\tau, T)$ correlators up to the midpoint, the corresponding covariance matrices of these data sets will be provided online [46]. Due to the use of the conserved-local current $G_{00}(\tau, T)$ is constant to very high accuracy and can be inferred from $\chi_{s} / T^{2}$ in Tab. $\mathrm{V}$.

[1] N. Brambilla, S. Eidelman, P. Foka, S. Gardner, A.S. Kronfeld, et al., "QCD and Strongly Coupled Gauge Theories: Challenges and Perspectives," Eur.Phys.J. C74, 2981 (2014), arXiv:1404.3723 [hep$\mathrm{ph}]$,

[2] Y. Aoki, G. Endrodi, Z. Fodor, S.D. Katz, and K.K. Szabo, "The Order of the quantum chromodynamics transition predicted by the standard model of particle physics," Nature 443, 675-678 (2006), arXiv:heplat/0611014 [hep-lat]

[3] Frank Geurts (STAR), "The STAR Dilepton Physics Program," Nucl.Phys. A904-905, 217c-224c (2013), arXiv:1210.5549 [nucl-ex].

[4] Harvey B. Meyer, "Transport Properties of the Quark-Gluon Plasma: A Lattice QCD Perspective," Eur.Phys.J. A47, 86 (2011), arXiv:1104.3708 [hep-lat],

[5] P. Braun-Munzinger, B. Friman, F. Karsch, K. Redlich, and V. Skokov, "Net-charge probability distributions in heavy ion collisions at chemical freeze-out," Nucl.Phys. A880, 48-64 (2012), arXiv:1111.5063 [hep-ph].

[6] J. Stachel, A. Andronic, P. Braun-Munzinger, and K. Redlich, "Confronting LHC data with the statistical hadronization model," J.Phys.Conf.Ser. 509, 012019 (2014), arXiv:1311.4662 [nucl-th],

[7] A. Bazavov et al. (HotQCD Collaboration), "Fluctuations and Correlations of net baryon number, electric charge, and strangeness: A comparison of lattice QCD results with the hadron resonance gas model," Phys.Rev. D86, 034509 (2012), arXiv:1203.0784 [hep-lat],

[8] Szabocls Borsanyi, Zoltan Fodor, Christian Hoelbling, Sandor D. Katz, Stefan Krieg, et al., "Full result for the QCD equation of state with 2+1 flavors," Phys.Lett. B730, 99-104 (2014), arXiv:1309.5258 [hep-lat]. 


\begin{tabular}{|c|c|c|c|c|c|c|c|c|c|}
\hline \multicolumn{10}{|c|}{$64^{3} \times 128, T=32(6) \mathrm{MeV}$} \\
\hline$\tau$ & $G_{i i}(\tau, T)$ & $\delta G_{i i}(\tau, T)$ & $\tau G_{i i}(\tau, T)$ & $\delta G_{i i}(\tau, T)$ & $\tau$ & $G_{i i}(\tau, T)$ & $\delta G_{i i}(\tau, T)$ & $\tau G_{i i}(\tau, T)$ & $\delta G_{i i}(\tau, T)$ \\
\hline 0 & $.6350 \mathrm{e}-01$ & $2.998 \mathrm{e}-04$ & 163. & $54.461 \mathrm{e}-$ & 32 & -06 & $1.412 \mathrm{e}-07$ & $481.1428 \mathrm{e}-08$ & -08 \\
\hline 1 & $5.2587 \mathrm{e}-02$ & $1.092 \mathrm{e}-04$ & 172.9 & $54.042 \mathrm{e}-07$ & & 06 & $1.344 \mathrm{e}-07$ & 491.8 & $4.908 \mathrm{e}-08$ \\
\hline 2 & $1.6643 \mathrm{e}-02$ & $3.171 \mathrm{e}-05$ & 182.3 & 53.7 & & & & & 4.8 \\
\hline 3 & $5.9285 \mathrm{e}-03$ & $1.084 \mathrm{e}-05$ & 191.8 & 53.5 & 5 & & $1.177 \mathrm{e}-07$ & 515. & -08 \\
\hline 4 & $2.6076 \mathrm{e}-03$ & 6 & 201. & & & & & & \\
\hline 5 & $1.3495 \mathrm{e}-03$ & $3.332 \mathrm{e}-06$ & 211.19 & 52.98 & 37 & 3.9 & $1.028 \mathrm{e}-07$ & 539 & 4.45 \\
\hline 6 & 7.859 & 2.20 & 229.6 & & & & & & \\
\hline 7 & $4.9835 \mathrm{e}-04$ & $1.561 \mathrm{e}-06$ & $237.8557 \mathrm{e}-06$ & $2.564 \mathrm{e}-07$ & 39 & 2.277 & $8.799 \mathrm{e}-08$ & 553.99 & $4.336 \mathrm{e}-08$ \\
\hline 8 & $3.3602 \mathrm{e}-04$ & $1.192 \mathrm{e}-06$ & 246.39 & 52.46 & & & $8.136 \mathrm{e}-08$ & 565.7 & e-08 \\
\hline 9 & $2.3710 \mathrm{e}-04$ & $9.685 \mathrm{e}-07$ & 255.23 & 62.3 & 41 & 07 & $7.643 \mathrm{e}-08$ & 576. & $4.223 \mathrm{e}-08$ \\
\hline 10 & $1.7282 \mathrm{e}-04$ & $8.212 \mathrm{e}-07$ & 264.31 & & & & $7.222 \mathrm{e}-08$ & 587.7 & $4.250 \mathrm{e}-08$ \\
\hline & $1.2917 \mathrm{e}-04$ & $7.202 \mathrm{e}-07$ & $273.5265 \mathrm{e}-06$ & $62.007 \mathrm{e}-07$ & 43 & 7.4499 & $6.713 \mathrm{e}-08$ & $598.4635 \mathrm{e}-08$ & $4.303 \mathrm{e}-08$ \\
\hline 12 & $9.8227 \mathrm{e}-05$ & $6.312 \mathrm{e}-07$ & $282.8993 \mathrm{e}-06$ & 6 1.880e-07 & & 5.1063 & $6.236 \mathrm{e}-08$ & $609.2662 \mathrm{e}-08$ & $4.395 \mathrm{e}-08$ \\
\hline 13 & 7.5773e-05 & $5.802 \mathrm{e}-07$ & $292.3682 \mathrm{e}-06$ & 6 1.725e-07 & 45 & $4.1019 \mathrm{e}-08$ & $5.864 \mathrm{e}-08$ & 61 1.0136e-07 & $4.602 \mathrm{e}-08$ \\
\hline 14 & $5.9029 \mathrm{e}-05$ & $5.481 \mathrm{e}-07$ & $301.9094 \mathrm{e}-06$ & 6 1.581e-07 & & $2.3223 \mathrm{e}-08$ & $5.499 \mathrm{e}-08$ & $629.7743 \mathrm{e}-08$ & $4.693 \mathrm{e}-08$ \\
\hline & $4.6229 \mathrm{e}-05$ & $4.982 \mathrm{e}-07$ & $31 \quad 1.5624 \mathrm{e}-06$ & 6 1.507e-07 & & $1.4130 \mathrm{e}-0$ & 5.22 & $\begin{array}{ll}63 & 9.7347 \mathrm{e}-08 \\
64 & 9.7577 \mathrm{e}-08\end{array}$ & $\begin{array}{l}4.820 \mathrm{e}-08 \\
4.932 \mathrm{e}-08\end{array}$ \\
\hline
\end{tabular}

TABLE VII. The vacuum vector correlators with ensemble parameters $64^{3} \times 128, T=32(6) \mathrm{MeV}, N_{s r c}=16$, $m_{\pi} \simeq 270 \mathrm{MeV}$ and $m_{\pi} L=4.2$.

\begin{tabular}{|c|c|c|c|c|c|c|c|c|c|}
\hline \multicolumn{3}{|c|}{$64^{3} \times 24, T=169(3) \mathrm{MeV}$} & \multicolumn{7}{|c|}{$64^{3} \times 20, T=203(4) \mathrm{MeV}\left|64^{3} \times 16, T=254(4) \mathrm{MeV}\right| 64^{3} \times 12, T=338(5) \mathrm{MeV}$} \\
\hline$\tau$ & $G_{i i}(\tau, T)$ & $\delta G_{i i}(\tau, T)$ & $\tau$ & $G_{i i}(\tau, T)$ & $\delta G_{i i}(\tau, T)$ & $\tau G_{i i}(\tau, T)$ & $\delta G_{i i}(\tau, T)$ & $\tau G_{i i}(\tau, T)$ & $\delta G_{i i}(\tau, T)$ \\
\hline 0 & $-1.6399 \mathrm{e}-01$ & $1.163 \mathrm{e}-04$ & 0 & $-1.6430 \mathrm{e}-01$ & $1.014 \mathrm{e}-04$ & $0-1.6463 \mathrm{e}-01$ & $1.001 \mathrm{e}-04$ & $0-1.6477 \mathrm{e}-01$ & $1.084 \mathrm{e}-04$ \\
\hline 1 & $5.2705 \mathrm{e}-02$ & $4.293 \mathrm{e}-05$ & 1 & $5.2719 \mathrm{e}-02$ & $3.575 \mathrm{e}-05$ & $15.2776 \mathrm{e}-02$ & $3.694 \mathrm{e}-05$ & $15.2798 \mathrm{e}-02$ & $4.143 \mathrm{e}-05$ \\
\hline 2 & $1.6667 \mathrm{e}-02$ & $1.277 \mathrm{e}-05$ & 2 & $1.6692 \mathrm{e}-02$ & $1.311 \mathrm{e}-05$ & $21.6768 \mathrm{e}-02$ & $1.045 \mathrm{e}-05$ & $21.6963 \mathrm{e}-02$ & $1.160 \mathrm{e}-05$ \\
\hline 3 & $5.9540 \mathrm{e}-03$ & $5.149 \mathrm{e}-06$ & 3 & $5.9975 \mathrm{e}-03$ & $4.781 \mathrm{e}-06$ & $36.0897 \mathrm{e}-03$ & $4.161 \mathrm{e}-06$ & $36.3814 \mathrm{e}-03$ & $4.744 \mathrm{e}-06$ \\
\hline 4 & $2.6307 \mathrm{e}-03$ & $2.711 \mathrm{e}-06$ & 4 & $2.6814 \mathrm{e}-03$ & $2.401 \mathrm{e}-06$ & $42.7971 \mathrm{e}-03$ & $2.282 \mathrm{e}-06$ & $43.1940 \mathrm{e}-03$ & $2.763 \mathrm{e}-06$ \\
\hline 5 & $1.3775 \mathrm{e}-03$ & $1.725 \mathrm{e}-06$ & 5 & $1.4382 \mathrm{e}-03$ & $1.515 \mathrm{e}-06$ & $51.5797 \mathrm{e}-03$ & $1.528 \mathrm{e}-06$ & $52.1229 \mathrm{e}-03$ & $2.166 \mathrm{e}-06$ \\
\hline 6 & $8.1961 \mathrm{e}-04$ & $1.262 \mathrm{e}-06$ & 6 & $8.9125 \mathrm{e}-04$ & $1.140 \mathrm{e}-06$ & $61.0665 \mathrm{e}-03$ & $1.197 \mathrm{e}-06$ & 6 1.8551e-03 & $2.063 \mathrm{e}-06$ \\
\hline 7 & $5.3822 \mathrm{e}-04$ & $1.076 \mathrm{e}-06$ & 7 & $6.2263 \mathrm{e}-04$ & $9.765 \mathrm{e}-07$ & $78.4697 \mathrm{e}-04$ & $1.106 \mathrm{e}-06$ & & \\
\hline 8 & $3.8362 \mathrm{e}-04$ & $9.826 \mathrm{e}-07$ & 8 & $4.8443 \mathrm{e}-04$ & $8.882 \mathrm{e}-07$ & $87.8483 \mathrm{e}-04$ & $1.188 \mathrm{e}-06$ & & \\
\hline 9 & $2.9444 \mathrm{e}-04$ & $9.473 \mathrm{e}-07$ & 9 & $4.1733 \mathrm{e}-04$ & $8.890 \mathrm{e}-07$ & & & & \\
\hline 10 & $2.4309 \mathrm{e}-04$ & $9.308 \mathrm{e}-07$ & & $3.9737 \mathrm{e}-04$ & $8.967 \mathrm{e}-07$ & & & & \\
\hline 11 & $2.1627 \mathrm{e}-04$ & $9.299 \mathrm{e}-07$ & & & & & & & \\
\hline 12 & $2.0807 \mathrm{e}-04$ & $9.329 \mathrm{e}-07$ & & & & & & & \\
\hline
\end{tabular}

TABLE VIII. The thermal vector correlators on the lattice sizes $64^{3} \times 24,20,16$ and 12 using the fixed scale approach.

[9] Szabolcs Borsanyi, Zoltan Fodor, Sandor D. Katz, Stefan Krieg, Claudia Ratti, et al., "Fluctuations of conserved charges at finite temperature from lattice QCD," JHEP 1201, 138 (2012), arXiv:1112.4416 [hep-lat] 
[10] Bastian B. Brandt, Anthony Francis, Harvey B. Meyer, and Daniel Robaina, "Chiral dynamics in the low-temperature phase of QCD," Phys.Rev. D90, 054509 (2014), arXiv:1406.5602 [hep-lat],

[11] Bastian B. Brandt, Anthony Francis, Harvey B. Meyer, and Daniel Robaina, "Pion quasiparticle in the low-temperature phase of QCD," Phys. Rev. D92, 094510 (2015), arXiv:1506.05732 [hep-lat],

[12] Jorge Casalderrey-Solana, Hong Liu, David Mateos, Krishna Rajagopal, and Urs Achim Wiedemann, "Gauge/String Duality, Hot QCD and Heavy Ion Collisions," (2011), arXiv:1101.0618 [hep-th],

[13] Gert Aarts, Chris Allton, Justin Foley, Simon Hands, and Seyong Kim, "Spectral functions at small energies and the electrical conductivity in hot, quenched lattice QCD," Phys.Rev.Lett. 99, 022002 (2007) arXiv:hep-lat/0703008 [HEP-LAT].

[14] Harvey B. Meyer, "A calculation of the shear viscosity in SU(3) gluodynamics," Phys. Rev. D76, 101701 (2007), arXiv:0704.1801 [hep-lat]

[15] A. Francis and O. Kaczmarek, "On the temperature dependence of the electrical conductivity in hot quenched lattice QCD," Prog.Part.Nucl.Phys. 67, 212-217 (2012), arXiv:1112.4802 [hep-lat]

[16] H.-T. Ding, A. Francis, O. Kaczmarek, F. Karsch, E. Laermann, et al., "Thermal dilepton rate and electrical conductivity: An analysis of vector current correlation functions in quenched lattice QCD," Phys.Rev. D83, 034504 (2011), arXiv:1012.4963 [hep-lat].

[17] O. Kaczmarek, E. Laermann, M. Müller, F. Karsch, H.T. Ding, et al., "Thermal dilepton rates from quenched lattice QCD," PoS ConfinementX, 185 (2012), arXiv:1301.7436 [hep-lat].

[18] Heng-Tong Ding, Olaf Kaczmarek, and Florian Meyer, "Vector spectral functions and transport properties in quenched QCD," (2014), arXiv:1412.5869 [hep-lat]

[19] Alessandro Amato, Gert Aarts, Chris Allton, Pietro Giudice, Simon Hands, et al., "Electrical conductivity of the quark-gluon plasma across the deconfinement transition," Phys.Rev.Lett. 111, 172001 (2013), arXiv:1307.6763 [hep-lat]

[20] Bastian B. Brandt, Anthony Francis, Harvey B. Meyer, and Hartmut Wittig, "Thermal Correlators in the $\rho$ channel of two-flavor QCD," JHEP 1303, 100 (2013), arXiv:1212.4200 [hep-lat].

[21] G. Backus and F. Gilbert, "The resolving power of gross Earth data," Geophys.J.R.Astron.Soc. 16, 169-205 (1968).

[22] Paul M. Hohler and Ralf Rapp, "Is $\rho$-Meson Melting Compatible with Chiral Restoration?" Phys.Lett. B731, 103-109 (2014), arXiv:1311.2921 [hep-ph].

[23] Harvey B. Meyer, "The Bulk Channel in Thermal Gauge Theories," JHEP 1004, 099 (2010), arXiv:1002.3343 [hep-lat].

[24] Anthony Francis, Benjamin Jaeger, Harvey B. Meyer, and Hartmut Wittig, "A new representation of the Adler function for lattice QCD," Phys.Rev. D88, 054502 (2013), arXiv:1306.2532 [hep-lat].

[25] Anthony Francis, Vera Guelpers, Benjamin Jaeger, Harvey Meyer, Georg von Hippel, et al., "The leading disconnected contribution to the anomalous magnetic moment of the muon," PoS LATTICE2014, 128 (2014), arXiv:1411.7592 [hep-lat],

[26] David Bernecker and Harvey B. Meyer, "Vector Correlators in Lattice QCD: Methods and applications," Eur.Phys.J. A47, 148 (2011), arXiv:1107.4388 [hep-lat].

[27] P.A. Baikov, K.G. Chetyrkin, J.H. Kuhn, and J. Rittinger, "Vector Correlator in Massless QCD at Order $O\left(\alpha_{s}^{4}\right)$ and the QED beta-function at Five Loop," JHEP 1207, 017 (2012), arXiv:1206.1284 $[$ hep-ph].

[28] K.G. Chetyrkin, R. Harlander, Johann H. Kuhn, and M. Steinhauser, "Mass corrections to the vector current correlator," Nucl.Phys. B503, 339-353 (1997), arXiv:hep-ph/9704222 [hep-ph].

[29] Peter Petreczky and Derek Teaney, "Heavy quark diffusion from the lattice," Phys.Rev. D73, 014508 (2006), arXiv:hep-ph/0507318 [hep-ph].

[30] S. Capitani, M. Della Morte, D. Djukanovic, G. von Hippel, J. Hua, B. Jaeger, B. Knippschild, H. B. Meyer, T. D. Rae, and H. Wittig, "Nucleon electromagnetic form factors in two-flavor QCD," Phys. Rev. D92, 054511 (2015), arXiv:1504.04628 [hep-lat],

[31] Kenneth G. Wilson, "Confinement of Quarks," Phys.Rev. D10, 2445-2459 (1974).

[32] Karl Jansen and Rainer Sommer (ALPHA collaboration), "O(alpha) improvement of lattice QCD with two flavors of Wilson quarks," Nucl.Phys. B530, 185-203 (1998), arXiv:hep-lat/9803017 [hep-lat].

[33] Martin Hasenbusch, "Speeding up the hybrid Monte Carlo algorithm for dynamical fermions," Phys.Lett. B519, 177-182 (2001), arXiv:hep-lat/0107019 [hep-lat].

[34] M. Hasenbusch and K. Jansen, "Speeding up lattice QCD simulations with clover improved Wilson fermions," Nucl.Phys. B659, 299-320 (2003), arXiv:hep-lat/0211042 [hep-lat]. 
[35] Marina Marinkovic and Stefan Schaefer, "Comparison of the mass preconditioned HMC and the DDHMC algorithm for two-flavour QCD," PoS LATTICE2010, 031 (2010), arXiv:1011.0911 [hep-lat].

[36] Martin Lüscher, "Schwarz-preconditioned HMC algorithm for two-flavour lattice QCD," Comput.Phys.Commun. 165, 199-220 (2005), http://luscher.web.cern.ch/luscher/DD-HMC/, arXiv:heplat/0409106 [hep-lat].

[37] Patrick Fritzsch, Francesco Knechtli, Bjorn Leder, Marina Marinkovic, Stefan Schaefer, et al., "The strange quark mass and Lambda parameter of two flavor QCD," Nucl.Phys. B865, 397-429 (2012). arXiv:1205.5380 [hep-lat].

[38] Bastian B. Brandt, Anthony Francis, Harvey B. Meyer, Hartmut Wittig, and Owe Philipsen, "QCD thermodynamics with two flavours of Wilson fermions on large lattices," PoS LATTICE2012, 073 (2012), arXiv:1210.6972 [hep-lat]

[39] Michele Della Morte, Roland Hoffmann, Francesco Knechtli, Rainer Sommer, and Ulli Wolff, "Nonperturbative renormalization of the axial current with dynamical Wilson fermions," JHEP 0507, 007 (2005), arXiv:hep-lat/0505026 [hep-lat].

[40] Bastian B. Brandt, Anthony Francis, and Harvey B. Meyer, "Antiscreening of the Ampère force in QED and QCD plasmas," Phys.Rev. D89, 034506 (2014), arXiv:1310.5160 [hep-lat].

[41] Bastian B. Brandt, Anthony Francis, Harvey B. Meyer, and Hartmut Wittig, "Two-flavour lattice QCD correlation functions in the deconfinement transition region," PoS ConfinementX, 186 (2012), arXiv:1302.0675 [hep-lat].

[42] B.B. Brandt, A. Francis, M. Laine, and H.B. Meyer, "A relation between screening masses and real-time rates," JHEP 1405, 117 (2014), arXiv:1404.2404 [hep-ph].

[43] Bastian B. Brandt, Anthony Francis, Harvey B. Meyer, Owe Philipsen, and Hartmut Wittig, " $Q C D$ thermodynamics with $O(a)$ improved Wilson fermions at $N_{f}=2$," PoS LATTICE2013, 162 (2014), arXiv:1310.8326 [hep-lat].

[44] Martin Lüscher, Stefan Sint, Rainer Sommer, and Peter Weisz, "Chiral symmetry and O(a) improvement in lattice QCD," Nucl.Phys. B478, 365-400 (1996), arXiv:hep-lat/9605038 [hep-lat].

[45] Stefan Sint and Peter Weisz, "Further results on $O(a)$ improved lattice QCD to one loop order of perturbation theory," Nucl.Phys. B502, 251-268 (1997), arXiv:hep-lat/9704001 [hep-lat].

[46] Our covariance matrices can be found at, "http://arxiv.org,".

[47] M. Asakawa, T. Hatsuda, and Y. Nakahara, "Maximum entropy analysis of the spectral functions in lattice QCD," Prog.Part.Nucl.Phys. 46, 459-508 (2001), arXiv:hep-lat/0011040 [hep-lat]

[48] I. Wetzorke, F. Karsch, E. Laermann, P. Petreczky, and S. Stickan, "Meson spectral functions at finite temperature," Nucl.Phys.Proc.Suppl. 106, 510-512 (2002), arXiv:hep-lat/0110132 [hep-lat],

[49] Yannis Burnier and Alexander Rothkopf, "Bayesian Approach to Spectral Function Reconstruction for Euclidean Quantum Field Theories," Phys.Rev.Lett. 111, 182003 (2013), arXiv:1307.6106 [hep-lat]

[50] F. Karsch and H.W. Wyld, "Thermal Green's Functions and Transport Coefficients on the Lattice," Phys.Rev. D35, 2518 (1987)

[51] Y. Burnier, M. Laine, and L. Mether, "A Test on analytic continuation of thermal imaginary-time data," Eur.Phys.J. C71, 1619 (2011), arXiv:1101.5534 [hep-lat].

[52] Y. Burnier and M. Laine, "Towards flavour diffusion coefficient and electrical conductivity without ultraviolet contamination," Eur.Phys.J. C72, 1902 (2012), arXiv:1201.1994 [hep-lat]

[53] Robert C. Myers, Andrei O. Starinets, and Rowan M. Thomson, "Holographic spectral functions and diffusion constants for fundamental matter," JHEP 11, 091 (2007), arXiv:0706.0162 [hep-th]

[54] William H. Press, Saul A. Teukolsky, William T. Vetterling, and Brian P. Flannery, "Numerical Recipes: The Art of Scientific Computing," Cambridge, UK: Univ. Pr. (2007).

[55] Harvey B. Meyer, "A calculation of the bulk viscosity in SU(3) gluodynamics," Phys. Rev. Lett. 100, 162001 (2008), arXiv:0710.3717 [hep-lat],

[56] J. Engels and O. Vogt, "Longitudinal and transverse spectral functions in the three-dimensional O(4) model," Nucl.Phys. B832, 538-566 (2010), arXiv:0911.1939 [hep-lat]

[57] H.T. Ding, A. Francis, O. Kaczmarek, F. Karsch, H. Satz, et al., "Charmonium properties in hot quenched lattice QCD," Phys.Rev. D86, 014509 (2012), arXiv:1204.4945 [hep-lat],

[58] K. Nakamura et al. (Particle Data Group), "Review of particle physics," J.Phys. G37, 075021 (2010).

[59] A. Schenk, "Pion propagation at finite temperature," Phys.Rev. D47, 5138-5155 (1993).

[60] Szabolcs Borsanyi et al., "Is there still any Tc mystery in lattice QCD? Results with physical masses in the continuum limit III," JHEP 09, 073 (2010), arXiv:1005.3508 [hep-lat], 
[61] A. Bazavov, T. Bhattacharya, M. Cheng, C. DeTar, H.T. Ding, et al., "The chiral and deconfinement aspects of the QCD transition," Phys.Rev. D85, 054503 (2012), arXiv:1111.1710 [hep-lat]. 